\title{
Exact Simulation of Truncated Lévy Subordinator
}

\author{
Angelos Dassios* \\ Jia Wei $\operatorname{Lim}^{\dagger}$ \\ London School of Economics \\ Brunel University London \\ Yan $\mathrm{Qu}^{\ddagger}$ \\ University of Warwick
}

15th October 2019

\begin{abstract}
A truncated Lévy subordinator is a Lévy subordinator in $\mathbb{R}^{+}$with Lévy measure restricted from above by a certain level $b$. In this paper, we study the path and distribution properties of this type of processes in detail and set up an exact simulation framework based on a marked renewal process. In particular, we focus on a typical specification of truncated Lévy subordinator, namely the truncated stable process. We establish an exact simulation algorithm for the truncated stable process, which is very accurate and efficient. Compared to the existing algorithm suggested in Chi (2012), our algorithm outperforms over all parameter settings. Using a distribution decomposition technique, we also develop an exact simulation algorithm for the truncated tempered stable process and other related processes. We illustrate an application of our algorithm as a valuation tool for stochastic hyperbolic discounting, and numerical analysis are provided to demonstrate the accuracy and effectiveness of our methods. We also show that variations of the result can also be used to sample two-sided truncated Lévy processes, two-sided Lévy processes via subordinating Brownian motions, and truncated Lévy driven Ornstein-Uhlenbeck processes.
\end{abstract}

Keywords: Monte Carlo simulation; Exact simulation; Marked Renewal Representation; Stable process; Truncated stable process; Truncated tempered stable process; Two-sided truncated Lévy process; Brownian motion subordination; Lévy driven Ornstein-Uhlenbeck process

\footnotetext{
*Department of Statistics, London School of Economics, London WC2A 2AE, United Kingdom. Email: a.dassios@1se.ac.uk

†Department of Mathematics, Brunel University London, Uxbridge UB8 3PH, United Kingdom. Email: jiawei.lim@brunel.ac.uk

${ }^{\ddagger}$ Department of Statistics, University of Warwick, Coventry CV4 7AL, United Kingdom. Email: y.qu3@1se.ac.uk
} 


\section{Introduction}

Lévy subordinators are real-valued Lévy processes with non-decreasing sample paths and common examples of Lévy subordinators are the Inverse Gaussian and Gamma processes. Truncated Lévy subordinators are Lévy subordinators which have jump sizes restricted to be below a certain level $b$. Just like the original non-truncated subordinator, these processes have bounded variation and infinite activity. They have many potential applications in finance and insurance. For instance, Lévy subordinators have been widely used to model aggregate claim processes in the literature, see Bertoin and Doney (1994); Furrer et al. (1997); Furrer (1998); Schmidli (2001); Morales and Schoutens (2003); Klüppelberg et al. (2004). However, in practice, truncated Lévy subordinators provide better representations of aggregate claims than non-truncated Lévy subordinators as there is often an upper limit to the liability coverage of most insurance policies and excess-of-loss reinsurance taken up by the insurer also ensure that claim liabilities do not exceed a certain limit. Besides practical applications in insurance, truncated Lévy subordinators are also closely related to the present value of stochastic perpetuities. Stochastic perpetuities are of interest in many fields including finance, astrophysics and number theory (see Vervaat (1979); Embrechts and Goldie (1994); Blanchet and Sigman (2011)). In particular, the value of a generalised Vervaat perpetuity can be distributionally decomposed into a truncated Gamma process and a compound Poisson process (see Dassios et al. (2019)). As an application, we show how the truncated stable process is related to the valuation of perpetuities with stochastic hyperbolic discounting. This form of discounting is used frequently in behavioural economics and environmental science, see Sozou (1998); Hepburn et al. (2010); Wang (2017).

Computational tools for Lévy related processes are very useful, since analytical and probability results are often difficult to obtain. While truncated Lévy processes have Lévy measure restricted to the interval $(0, b)$, their density has support on the whole positive real line. For most truncated Lévy processes, this density is unknown or only has an infinite series representation. Hence, it is difficult to find an exact simulation scheme to sample this type of processes at a fixed time $t$. Approximations can be employed such as that described in Asmussen and Rosiński (2001), but it imposes a trade-off between computing effort and approximation error. Chi (2012) proposed an approach by establishing integral series expansion of the density of truncated Lévy subordinator on $[b, \infty)$ in terms of its density on $(0, b)$ and applying rejection sampling suggested in Devroye (1968) to the integral series expansion to sample the truncated Lévy subordinator, but this is very computationally intensive. 
In this paper, we propose a general simulation framework to generate the position of truncated Lévy subordinator at a fixed time $t$, where no procedure of truncation, numerical inversion or discretisation is required. Our approach is based on a path decomposition of the process, into subsequent hitting times of level $b$. This can then be simulated using a marked renewal process the process increases $b$ units plus the associated overshoot at every hitting time of level $b$. Thus, in order to simulate the position of the process at a fixed time, we only need to know the joint distribution of the hitting time and overshoot, and the distribution of the process given the condition that the position of the process is less than the truncation level $b$. These distributions can be found for a wide range of truncated subordinators, in particular it can be derived from the density of the original non-truncated subordinator. Compared with Chi (2012), our procedure is achievable without the need to derive integral series expansion for the density of the truncated Lévy process. Another advantage of our method is that the pairs of random variables representing the hitting time and overshoots are independent and identically distributed, so our algorithm can be vectorised to reduce computation time.

For implementation, we focus on a typical specification of truncated Lévy subordinators, i.e. truncated stable process. We make use of the Zolotarev integral representation of the density of stable processes (Zolotarev, 1966), together with the aid of multi-dimensional acceptance-rejection schemes, to develop simulation algorithms to sample the hitting times, overshoots and position of the process given it is less than $b$. By generating these quantities within the marked renewal framework, we obtain a simulation algorithm to sample the position of the truncated stable process at a fixed time $t$. The algorithm is very accurate and efficient. Compared to the existing algorithm suggested in Chi (2012), our method outperforms over all parameter settings and even more substantially when the fixed time $t$ is large.

The rest of this paper will be structured as follows. In Section 2, we formally introduce the truncated Lévy process, and derive key results such as the joint density of its hitting time and overshoot. In Section 3, we illustrate the marked renewal representation for the truncated Lévy process and establish the exact simulation framework accordingly. In Section 4, we look at some specific examples of truncated Lévy subordinators. Based on the marked renewal framework, we derive exact simulation algorithms for the truncated stable process and truncated tempered stable process accordingly. Extensive numerical analysis and comparisons have been carried out and reported in detail, which demonstrate the accuracy and effectiveness of our algorithms. An application on the valuation of perpetuity with stochastic hyperbolic discounting is also provided. In Section 5, based on the marked renewal approach for truncated Lévy subordinator, we propose exact simula- 


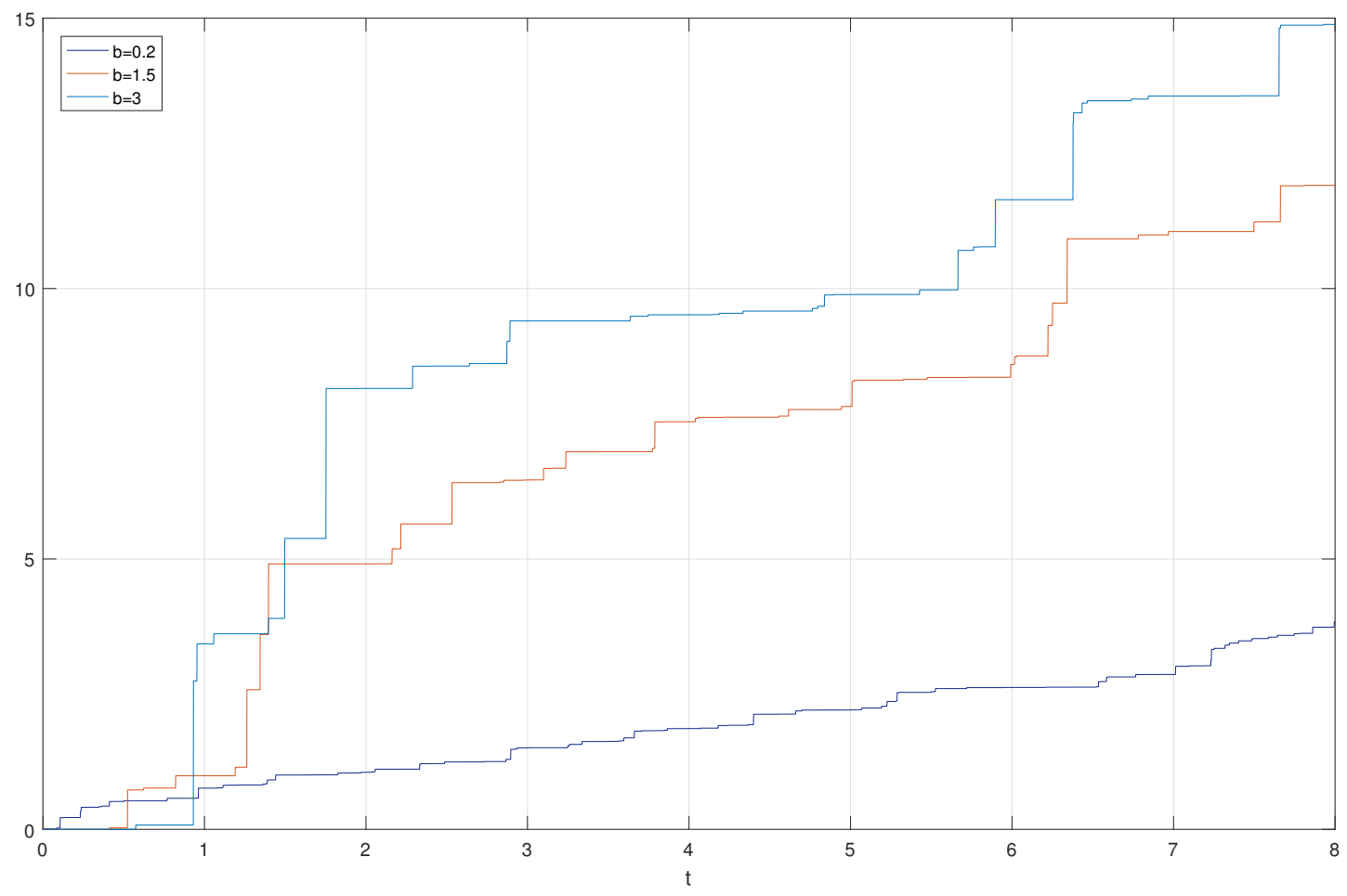

Figure 1: Sample paths of a truncated Lévy Subordinator with $b=0.2,1.5,3$, respectively.

tion schemes for two-sided truncated Lévy processes, two-sided Lévy processes via subordinating Brownian motions, and truncated Lévy driven Ornstein-Uhlenbeck models. Finally, in Section 6, we draw a brief conclusion for the paper.

\section{Definitions and Distributional Properties}

Definition 2.1. A truncated Lévy subordinator $Z_{t}$ is defined by restricting the support of the Lévy measure $\nu$ with an upper bound $b$. The Laplace transform of $Z_{t}$ is given as

$$
\mathbb{E}\left[e^{-v Z_{t}}\right]=\exp \left(-t \int_{0}^{b}\left(1-e^{-v z}\right) \nu(\mathrm{d} z)\right), \quad v \in \mathbb{R}^{+}
$$

$Z_{t}$ preserves most properties of the original Lévy subordinator. It is a non-decreasing process with bounded variation and infinite activity, i.e, there are infinite number of jumps over a compact interval. The only difference is that the sizes of those infinite number of jumps are not allowed to exceed a certain level $b$. This is illustrated Figure 1, where we plot the sample paths of a truncated Lévy subordinator $Z_{t}$ for three different truncation levels $b$. 
The original non-truncated Lévy subordinator $X_{t}$ is thus equivalent to

$$
X_{t} \stackrel{\mathcal{D}}{=} Z_{t}+R_{t}
$$

where $Z_{t}$ is the truncated Lévy subordinator with Lévy measure restricted to $(0, b)$, and $R_{t}$ is a compound Poisson process with mean $t \int_{b}^{\infty} \nu(\mathrm{d} x)$, independent from $Z_{t}$, and the density of its jump sizes is

$$
f(x)=\frac{\nu(\mathrm{d} x)}{\int_{b}^{\infty} \nu(\mathrm{d} x)}, \quad b \leq x<\infty .
$$

This allows us to consider subordinators whose Lévy measure is discontinuous at $b$, and thus jump sizes are not characterised by a single continuous distribution. More generally, $R_{t}$ can be replaced by any compound Poisson process with an arbitrary Poisson rate and jump distribution.

The paths of the truncated Lévy subordinator $Z_{t}$ can be characterised via hitting times and associated overshoots. Let $T$ be the first hitting time of level $b$ of the truncated Lévy subordinator $Z_{t}$, and $W$ be the associated overshoot at time $T$, i.e.

$$
\begin{aligned}
T: & =\inf \left\{t>0 \mid Z_{t}>b\right\} \\
W: & =Z_{T}-b .
\end{aligned}
$$

The general representation of the joint distribution of the first passage time and the associated overshoot is formulated in Theorem 2.1 below.

Theorem 2.1. Let $T$ be the first hitting time of level $b$ of $Z_{t}$ with Lévy measure $\nu$, and $W$ be the overshoot at time $T$. Then the joint probability density function of $(T, W)$ is given by

$$
f_{T, W}(t, w)=\int_{0}^{b} f_{Z_{t}}(z) \nu(b+w-z) \mathrm{d} z
$$

where $t \in(0, \infty), w \in(0, b)$, and $f_{Z_{t}}(z)$ denotes the density of $Z_{t}$ within $(0, b)$. In particular, we have

$$
f_{Z_{t}}(z)=e^{\bar{\nu}(b) t} f_{X_{t}}(z) \mathbf{1}_{\{0<z<b\}}
$$

where $\bar{\nu}(s):=\int_{s}^{\infty} \nu(x) \mathrm{d} x$, and $f_{X_{t}}(\cdot)$ is the density of $X_{t}$ with Laplace transform

$$
\mathbb{E}\left[e^{-v X_{t}}\right]=\exp \left(-t \int_{0}^{\infty}\left(1-e^{-v x}\right) \nu(\mathrm{d} x)\right)
$$


Proof. Using the strong Markov property of Lévy processes, we have

$$
\begin{aligned}
P(T \in d t, W>w) & =\lim _{\epsilon \rightarrow 0} \frac{1}{\epsilon} P\left(Z_{t-\epsilon} \leq b, Z_{t}>b+w\right) \\
& =\lim _{\epsilon \rightarrow 0} \frac{1}{\epsilon} \int_{0}^{b} P\left(Z_{t-\epsilon} \in \mathrm{d} z\right) P\left(Z_{\epsilon}>b+w-z\right) \\
& =\int_{0}^{b} f_{Z_{t}}(z) \int_{b+w-z}^{\infty} \nu(\mathrm{d} s) \mathrm{d} z
\end{aligned}
$$

with

$$
\begin{aligned}
\lim _{\epsilon \rightarrow 0} \frac{1}{\epsilon} P\left(Z_{\epsilon}>z\right) & =\lim _{\epsilon \rightarrow 0} \mathcal{L}^{-1}\left\{\frac{1}{\epsilon v}\left(1-\exp \left(-\epsilon \int_{0}^{\infty}\left(1-e^{-v s}\right) \nu(s) \mathrm{d} s\right)\right)\right\} \\
& =\mathcal{L}^{-1}\left\{\frac{1}{v} \int_{0}^{\infty}\left(1-e^{-v s}\right) \nu(s) \mathrm{d} s\right\} \\
& =\int_{z}^{\infty} \nu(s) \mathrm{d} s
\end{aligned}
$$

where $\mathcal{L}^{-1}$ denotes the inverse Laplace transform. Differentiating (2.6) with respect to $w$, the joint density of $(T, W)$ directly follows (2.3). The density of $Z_{t}$ within $(0, b)$ can be derived though its Laplace transform, we have

$$
\begin{aligned}
& f_{Z_{t}}(z) \\
= & \mathcal{L}^{-1}\left\{\mathbb{E}\left[e^{-v Z_{t}}\right]\right\} \mathbf{1}_{\{0<z<b\}} \\
= & \mathcal{L}^{-1}\left\{\exp \left(-t \int_{0}^{\infty}\left(1-e^{-v z}\right) \nu(\mathrm{d} z)\right) \exp \left(t \int_{b}^{\infty}\left(1-e^{-v z}\right) \nu(\mathrm{d} z)\right)\right\} \mathbf{1}_{\{0<z<b\}} \\
= & \mathcal{L}^{-1}\left\{\exp \left(-t \int_{0}^{\infty}\left(1-e^{-v z}\right) \nu(\mathrm{d} z)\right) \exp \left(t \int_{b}^{\infty} \nu(\mathrm{d} z)\right) \exp \left(-t \int_{b}^{\infty} e^{-v z} \nu(\mathrm{d} z)\right)\right\} \mathbf{1}_{\{0<z<b\}} \\
= & \mathcal{L}^{-1}\left\{\int_{0}^{\infty} e^{-v z} f_{X_{t}}(z) \mathrm{d} z \exp \left(t \int_{b}^{\infty} \nu(\mathrm{d} z)\right) \sum_{k=0}^{\infty} \frac{(-t)^{k}}{k !}\left(\int_{b}^{\infty} e^{-v z} \nu(\mathrm{d} z)\right)^{k} \mathbf{1}_{\{0<z<b\}}\right. \\
= & e^{\bar{\nu}(b) t} f_{X_{t}(z) \mathbf{1}_{\{0<z<b\}} .}
\end{aligned}
$$

where $f_{X_{t}}(\cdot)$ denotes the density of $X_{t}$ with Laplace transform (2.5).

Under the circumstance that the first passage time of $Z_{t}$ hits level $b$ is greater than $t$, the distribution of the truncated process $Z_{t}$ is characterised via its density within $(0, b)$. The details are illustrated in Theorem 2.2. 
Theorem 2.2. Given the hitting time $T$, the conditional density of $\left\{Z_{t} \mid T>t\right\}$ is given by

$$
f(x \mid T>t)=\frac{f_{X_{t}}(x)}{\int_{0}^{b} f_{X_{t}}(x) \mathrm{d} x}, \quad 0<x<b,
$$

where $f_{X_{t}}(\cdot)$ denotes the density of $X_{t}$ with Laplace transform (2.5).

Proof. We know that

$$
\left\{Z_{t} \mid T>t\right\} \stackrel{\mathcal{D}}{=}\left\{Z_{t} \mid Z_{t}<b\right\}
$$

since $\{T>t\}$ is equivalent to $\left\{Z_{t}<b\right\}$. The density therefore satisfies

$$
f(x \mid T>t)=f(x \mid x<b)=\frac{f_{X_{t}}(x)}{\int_{0}^{b} f_{X_{t}}(x) \mathrm{d} x} .
$$

Thus, the density immediately follows (2.7).

\section{Marked Renewal Representation}

The paths of the truncated subordinator $Z_{t}$ can be characterised by a marked renewal process. First, we define a sequence of hitting time spans $T_{1}, T_{2}, T_{3}, \ldots$, and denoting $S_{i}=\sum_{j=1}^{i} T_{j}$, let

$$
T_{i}:=\inf \left\{t>0 \mid Z_{S_{i-1}+t}>Z_{S_{i-1}}+b\right\}, \quad i=2,3, \ldots
$$

We further define $W_{1}, W_{2}, \ldots$ to be the overshoots at time $S_{1}, S_{2}, \ldots$, i.e.

$$
W_{i}:=Z_{S_{i}}-Z_{S_{i-1}}-b
$$

Hence, at time $S_{i}$ the process will automatically increase by $\left(b+W_{i}\right)$ units for all $i$. Since the process $Z_{t}$ has independent and stationary increments, each pair of $\left(T_{i}, W_{i}\right)$ are independent and identically distributed (i.i.d) with joint density given in Theorem 2.1. In addition, $W_{i}$ will be bounded by 0 and $b$ for all $i$ as the jump sizes of the process is restricted with an upper bound $b$. The value of the process at time $S_{n}$ will be $Z_{S_{n}}=\sum_{i=1}^{n}\left(b+W_{i}\right)$. The position of the process $Z_{t}$ at time $t$ therefore can be expressed as using a marked renewal process as follows,

$$
Z_{t}=\sum_{i=1}^{N_{t}}\left(b+W_{i}\right)+\left(Z_{t}-Z_{S_{N_{t}}} \mid S_{N_{t}}<t<S_{N_{t}}+T_{n+1}\right)
$$


Figure 2: Graphical illustration of a sample path of $X_{t}$

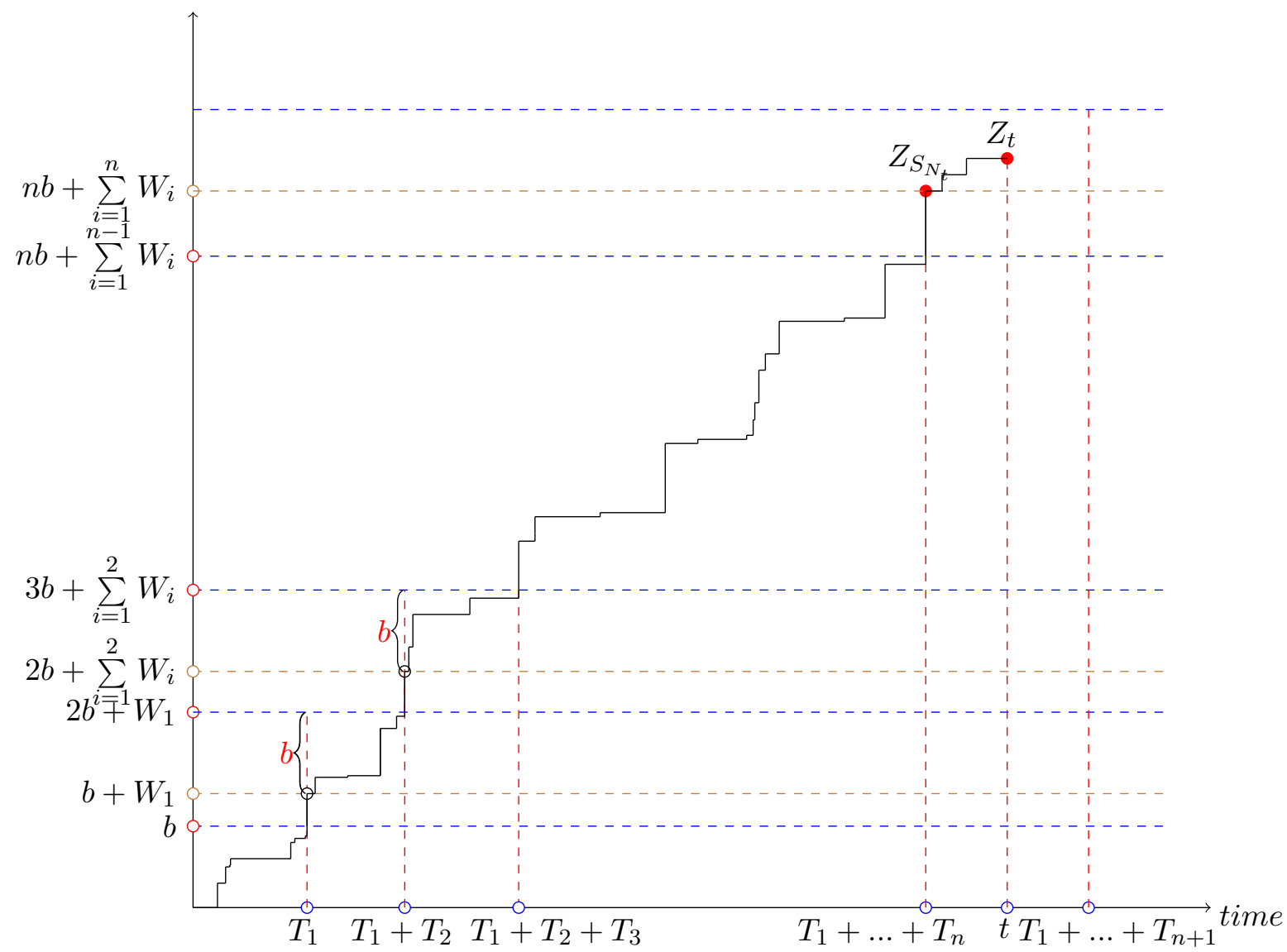

where $N_{t}=\sum_{i=1}^{\infty} \mathbf{1}_{\left\{S_{i} \leq t\right\}}$ is determined via (3.1) such that $t \in\left[S_{N_{t}}, S_{N_{t}}+T_{n+1}\right)$. We also use Figure 2 to illustrate the marked renewal idea graphically.

The first part in (3.3) represents the position of the truncated process at time $S_{N_{t}}$ before reaches $t$. The second term in (3.3) represents the movement of the process within the time span $t-S_{N_{t}}$ and we have

$$
\left\{Z_{t}-Z_{S_{N_{t}}} \mid S_{N_{t}}<t<S_{N_{t}}+T_{n+1}\right\} \stackrel{\mathcal{D}}{=}\left\{Z_{t-S_{N_{t}}} \mid t-S_{N_{t}}<T_{n+1}\right\}
$$

Conditioning on $t-S_{N_{t}}<T_{n+1}$, the distribution of $Z_{t-S_{N_{t}}}$ satisfies (2.7) in Theorem 2.2. Thus, $X_{t}$ can be simulated by generating pairs of hitting time and overshoot $\left(T_{i}, W_{i}\right)$, stopping when the sum of $T_{i}$ that have been generated, say $S_{N_{t}+1}$, becomes larger than the input $t$. We then generate the part $\left\{Z_{t-S_{N_{t}}} \mid t-S_{N_{t}}<T_{n+1}\right\}$ and return to (3.3). We give the details of the exact simulation method in Algorithm 3.1. In particular, we show how to emphasize the marked renewal procedure using a recursive loop. For implementation, one needs to specify the Lévy measure in explicit form in order to identify the joint density of $(T, W)$ and the conditional density of $\left\{Z_{t} \mid T>t\right\}$. In the following sections, we consider several typical examples of truncated Lévy subordinators and 


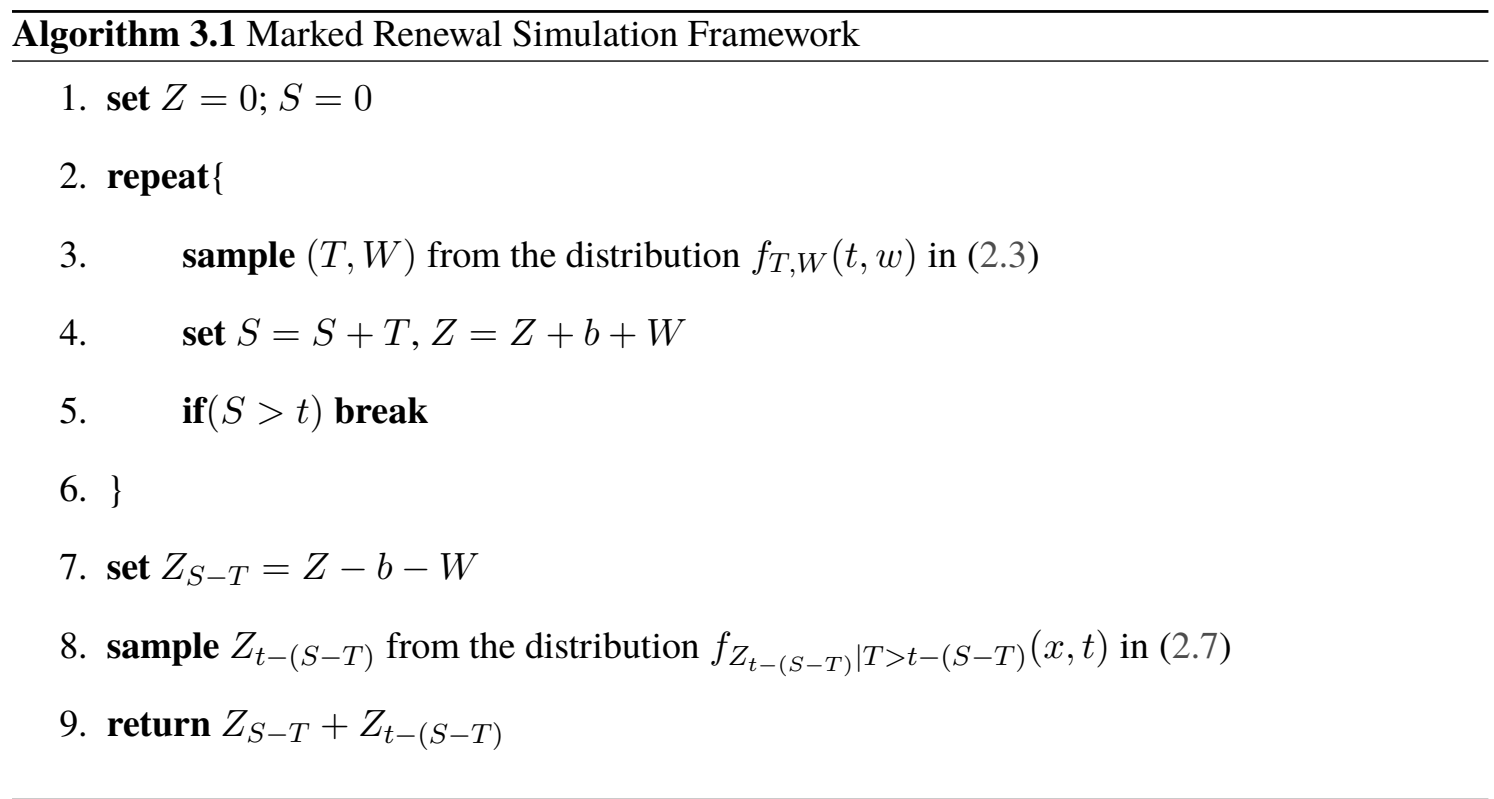

develop associated simulation algorithms based on Algorithm 3.1.

\section{Truncated Stable Process}

Truncated stable processes retain most properties of stable processes. In this section, we analyse distributional properties of these processes and develop corresponding exact simulation algorithms based on marked renewal framework.

\subsection{Definitions and Distributional Properties}

Let $Z_{t}$ be a truncated Lévy subordinator with Lévy measure

$$
\nu(\mathrm{d} z)=\frac{\alpha z^{-\alpha-1} e^{-\mu z}}{\Gamma(1-\alpha)} \mathbf{1}_{\{0<z<b\}} \mathrm{d} z, \quad \alpha \in(0,1), \quad \mu \in \mathbb{R}
$$

For $\mu=0, Z_{t}$ is a truncated stable process with stability $\alpha$, abbreviated as $T S(\alpha, t)$. For $\mu>0, Z_{t}$ is a truncated tempered stable process with stability parameter $\alpha$ and tilting parameter $\mu$, denoted by $\operatorname{TTS}(\alpha, \mu, t)$. For $\mu<0, Z_{t}$ is also a well-defined Lévy subordinator.

The Laplace transform of $Z_{t}$ can be expressed as

$$
\begin{aligned}
\mathbb{E}\left[e^{-v Z_{t}}\right] & =\exp \left(-\frac{\alpha t}{\Gamma(1-\alpha)} \int_{0}^{b}\left(1-e^{-v z}\right) \frac{e^{-\mu z}}{z^{\alpha+1}} \mathrm{~d} z\right) \\
& =\exp \left(-\frac{\alpha \hat{t}}{\Gamma(1-\alpha)} \int_{0}^{1}\left(1-e^{-v b z}\right) \frac{e^{-\mu b z}}{z^{\alpha+1}} \mathrm{~d} z\right)=\mathbb{E}\left[e^{-v b Z_{\hat{t}}}\right]
\end{aligned}
$$


where $\hat{t}=t b^{-\alpha}$. Hence, for $\mu=0$, the truncated stable $Z_{t}$ with truncation level $b$ at time $t$ is identical to $b Z_{\hat{t}}$, where $Z_{\hat{t}}$ is a truncated stable with truncation level 1 at time $\hat{t}$. For $\mu>0$, the truncated tempered stable with truncation level $b$, titling parameter $\mu$ at time $t$ is identical to $b Z_{\hat{t}}$, where $Z_{\hat{t}}$ is a truncated tempered stable with truncation level 1 , tilting parameter $\mu b$ at time $\hat{t}$. Therefore, without loss of generality, we set $b=1$ in here and other parts of the paper for simplicity.

As the density of a stable process can be expressed using an integral representation of Zolotarev (1966), see Kanter (1975), Zolotarev (1986), we can easily obtain analytical expression for the joint distribution $(T, W)$ and the distribution of $\left\{Z_{t} \mid T>t\right\}$ based on Theorem 2.1 and 2.2.

Lemma 4.1. For a truncated stable process $Z_{t}$ the joint probability density function of $(T, W)$ is given by

$f_{T, W}(t, w)=\int_{w}^{1} \int_{0}^{\pi} \frac{\alpha e^{\frac{t}{\Gamma(1-\alpha)}}}{\pi \Gamma(1-\alpha)} \frac{\alpha}{1-\alpha} A(u) y^{-\frac{1}{1-\alpha}} t^{\frac{1}{1-\alpha}} e^{-A(u) y^{-\frac{\alpha}{1-\alpha}} t^{\frac{1}{1-\alpha}}} \frac{1}{(1+w-y)^{\alpha+1}} \mathrm{~d} u \mathrm{~d} y$

and

$$
A(u):=\left[\frac{\sin (\alpha u)^{\alpha} \sin ((1-\alpha) u)^{1-\alpha}}{\sin u}\right]^{\frac{1}{1-\alpha}} .
$$

In addition, given the hitting time $T$, the conditional density of $\left\{Z_{t} \mid T>t\right\}$ is given by

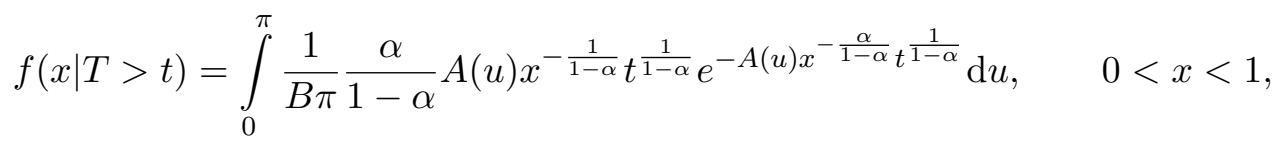

where

$$
B=\int_{0}^{\pi} \frac{1}{\pi} e^{-A(u) t^{\frac{1}{1-\alpha}}} \mathrm{d} u
$$

Proof. For a stable process $S_{t}$ with stability index $\alpha$ and Laplace transform $\mathbb{E}\left[e^{-v S_{t}}\right]=e^{-t v^{\alpha}}$, the density of $S_{t}$ is of the form

$$
\begin{aligned}
f_{\alpha}(x, t) & =t^{-\frac{1}{\alpha}} f_{\alpha}\left(t^{-\frac{1}{\alpha}} x, 1\right) \\
& =\frac{1}{\pi} \int_{0}^{\pi} \frac{\alpha}{1-\alpha} A(u) x^{-\frac{1}{1-\alpha}} t^{\frac{1}{1-\alpha}} e^{-A(u) x^{-\frac{\alpha}{1-\alpha}} t^{\frac{1}{1-\alpha}}} \mathrm{d} u
\end{aligned}
$$

with $A(u)$ defined in (4.3) (Devroye, 2009). Hence, the conditional density of $Z_{t}$ within $(0,1)$ is 
given by

$$
\begin{aligned}
f(x \mid T>t) & =\exp \left(\frac{t \alpha}{\Gamma(1-\alpha)} \int_{1}^{\infty} x^{-\alpha-1} \mathrm{~d} x\right) f_{\alpha}(x, t) \\
& =\frac{e^{\frac{t}{\Gamma(1-\alpha)}}}{\pi} \int_{0}^{\pi} \frac{\alpha}{1-\alpha} A(u) x^{-\frac{1}{1-\alpha}} t^{\frac{1}{1-\alpha}} e^{-A(u) x^{-\frac{\alpha}{1-\alpha}} t^{\frac{1}{1-\alpha}}} \mathrm{d} u .
\end{aligned}
$$

The joint probability density function of the hitting time and overshoot $(T, W)$ therefore directly follows (4.2).

For truncated tempered stable process, the joint probability density function of $(T, W)$ and the conditional density of $\left\{Z_{t} \mid T>t\right\}$ depends on the density of the associated tempered stable process. The details are given below in Lemma 4.2.

Lemma 4.2. For a truncated tempered stable process $Z_{t}$ with Lévy measure (4.1) for $\mu>0$, the density of $Z_{t}$ within $(0,1)$ is given as

$$
f_{Z_{t}}(x)=\frac{e^{\frac{t}{\Gamma(1-\alpha)}\left(e^{-\mu}+\mu^{\alpha} \gamma(1-\alpha, \mu)\right)}}{\pi} \int_{0}^{\pi} \frac{\alpha}{1-\alpha} A(u) x^{-\frac{1}{1-\alpha}} t^{\frac{1}{1-\alpha}} e^{-A(u) x^{-\frac{\alpha}{1-\alpha}} t^{\frac{1}{1-\alpha}}-\mu x} \mathrm{~d} u
$$

with $A(u)$ defined in (4.3) and $\gamma(\cdot, \cdot)$ is the lower incomplete gamma function such that

$$
\gamma(s, x)=\int_{0}^{x} y^{s-1} e^{-y} \mathrm{~d} y
$$

Proof. For a general tempered stable process $S_{t}$ with Laplace transform

$$
\mathbb{E}\left[e^{-v S_{t}}\right]=\exp \left(-t\left[(\mu+v)^{\alpha}-\mu^{\alpha}\right]\right)
$$

The density of $S_{t}$ is of the following form

$$
f_{\alpha, \mu}(x, t)=e^{\mu^{\alpha} t-\mu x} t^{-\frac{1}{\alpha}} f_{\alpha}\left(t^{-\frac{1}{\alpha}} x, 1\right) .
$$

where $f_{\alpha}(\cdot, 1)$ is the associated density function with stability $\alpha$. Hence we obtain (4.7) based on the Zolotarev integral representation and Theorem 2.1.

Lemma 4.3. For a truncated tempered stable process $X_{t}$, the joint probability density function of 
$(T, W)$ is of the form

$f_{T, W}(t, w)=\int_{w}^{1} \int_{0}^{\pi} \frac{\alpha e^{\frac{\left(e^{-\mu}+\mu^{\alpha} \gamma(1-\alpha, \mu)\right) t}{\Gamma(1-\alpha)}}}{\pi \Gamma(1-\alpha)} \frac{\alpha}{1-\alpha} A(u) y^{-\frac{1}{1-\alpha}} t^{\frac{1}{1-\alpha}} e^{-A(u) y^{-\frac{\alpha}{1-\alpha}} t^{\frac{1}{1-\alpha}}} \frac{e^{-\mu(1+w)}}{(1+w-y)^{\alpha+1}} \mathrm{~d} u \mathrm{~d} y$.

In addition, given the hitting, the conditional density of $\left\{Z_{t} \mid T>t\right\}$ is given by

$$
f(x \mid T>t)=\int_{0}^{\pi} \frac{1}{D \pi} \frac{\alpha}{1-\alpha} A(u) x^{-\frac{1}{1-\alpha}} t^{\frac{1}{1-\alpha}} e^{-A(u) x^{-\frac{\alpha}{1-\alpha}} t^{\frac{1}{1-\alpha}}-\mu x} \mathrm{~d} u
$$

where

$$
D=\int_{0}^{1} \int_{0}^{\pi} \frac{\alpha}{\pi(1-\alpha)} A(u) x^{-\frac{1}{1-\alpha}} t^{\frac{1}{1-\alpha}} e^{-A(u) x^{-\frac{\alpha}{1-\alpha}} t^{\frac{1}{1-\alpha}}-\mu x} \mathrm{~d} u \mathrm{~d} x .
$$

Proof. (4.8) and (4.9) are derived directly based on Theorem 2.1, 2.2 and Lemma 4.2.

\subsection{Exact Simulation Scheme}

For $\mu=0$, with analytical expressions for the joint probability density function $(T, W)$ and the conditional density of $\left\{Z_{t} \mid T>t\right\}$ in Lemma 4.1, we can develop simulation algorithms to sample $(T, W)$ and $\left\{Z_{t} \mid T>t\right\}$ accordingly. The details are provided in the following Algorithm 4.1 and 4.2 .

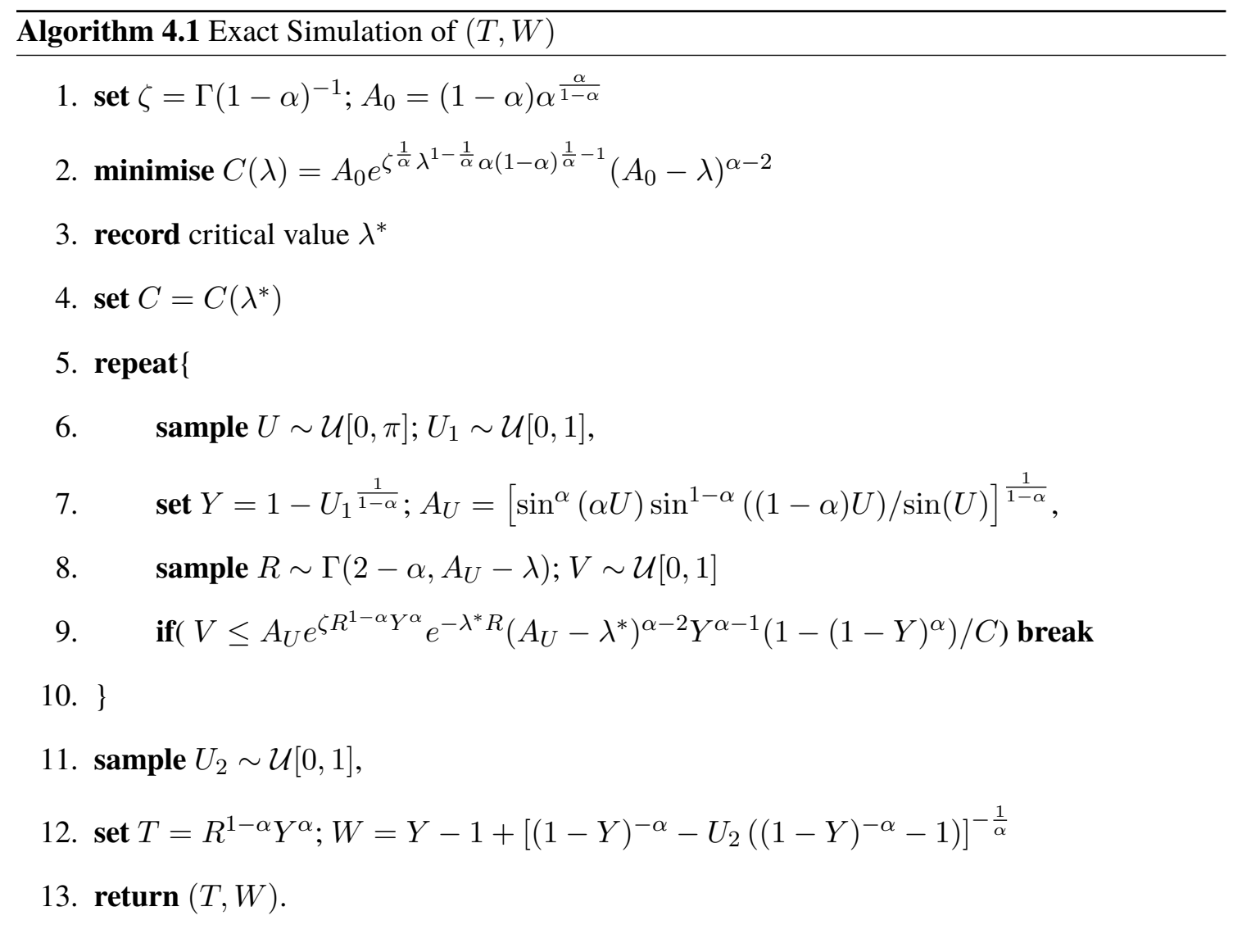


Proof. The simulation algorithm for $(T, W)$ is developed based on a multi-dimensional A/R scheme.

The joint probability density function of the hitting time and overshoot is given by

$$
f_{T, W}(t, w)=\int_{w}^{1} \int_{0}^{\pi} f(t, y, u) f(w \mid y) \mathrm{d} u \mathrm{~d} y,
$$

where

$$
f(t, y, u)=\frac{\alpha \zeta}{\pi(1-\alpha)} A(u) y^{-\frac{1}{1-\alpha}} t^{\frac{1}{1-\alpha}} e^{-A(u) y^{-\frac{\alpha}{1-\alpha}} t^{\frac{1}{1-\alpha}}+\zeta t}\left((1-y)^{-\alpha}-1\right)
$$

for $t \in(0, \infty), y \in(0,1), u \in(0, \pi)$, and

$$
f(w \mid y)=\frac{\frac{1}{(1+w-y)^{\alpha+1}}}{\frac{1}{\alpha}\left((1-y)^{-\alpha}-1\right)}, \quad 0<w<y .
$$

with $\zeta=\frac{1}{\Gamma(1-\alpha)}$. We then make a transformation by setting $r=y^{-\frac{\alpha}{1-\alpha}} t^{\frac{1}{1-\alpha}}$, the density of $(R, Y, U)$ is of the form

$$
f(r, y, u)=\frac{\alpha \zeta e^{\zeta r^{1-\alpha} y^{\alpha}}}{\pi} A(u) r^{(1-\alpha)} y^{-(1-\alpha)} e^{-A(u) r}(1-y)^{-\alpha}\left(1-(1-y)^{\alpha}\right),
$$

for $r \in(0, \infty), y \in(0,1), u \in(0, \pi)$.

To simulate $(R, Y, U)$, we choose an envelope $\left(R^{\prime}, Y^{\prime}, U^{\prime}\right)$ with density

$$
g(r, y, u)=\frac{1}{\pi} \frac{(A(u)-\lambda)^{2-\alpha} r^{1-\alpha} e^{-(A(u)-\lambda) r}}{\Gamma(2-\alpha)}(1-\alpha)(1-y)^{-\alpha},
$$

where $U^{\prime} \sim \mathcal{U}[0, \pi], R^{\prime} \sim \Gamma(2-\alpha, A(U)-\lambda)$, and $Y$ with CDF $G(y)=(1-y)^{1-\alpha}$, which can be inverted explicitly, i.e. $G^{-1}(u)=1-u^{\frac{1}{1-\alpha}}$. The random variable can be simulated via inverse transformation by setting $Y^{\prime}=1-U_{1}^{\frac{1}{1-\alpha}}$ with $U_{1} \sim \mathcal{U}[0,1]$. Hence, the ratio of these two densities is given as

$$
\begin{aligned}
\frac{f(r, y, u)}{g(r, y, u)} & =\frac{\alpha \zeta \Gamma(2-\alpha) A(u)}{(1-\alpha)(A(u)-\lambda)^{2-\alpha}} e^{\zeta r^{1-\alpha} y^{\alpha}} e^{-\lambda r} y^{\alpha-1}\left(1-(1-y)^{\alpha}\right) \\
& \leq \frac{\alpha \zeta \Gamma(2-\alpha) A_{0}}{(1-\alpha)\left(A_{0}-\lambda\right)^{2-\alpha}} e^{\zeta r^{1-\alpha} y^{\alpha}} e^{-\lambda r} y^{\alpha-1}\left(1-(1-y)^{\alpha}\right) \\
& \leq \frac{\alpha \zeta \Gamma(2-\alpha) A_{0}}{(1-\alpha)\left(A_{0}-\lambda\right)^{2-\alpha}} e^{\zeta^{\frac{1}{\alpha}} \lambda^{1-\frac{1}{\alpha}} \alpha(1-\alpha)^{\frac{1}{\alpha}-1}}=C(\lambda),
\end{aligned}
$$

where $A_{0}=(1-\alpha) \alpha^{\frac{\alpha}{1-\alpha}}$. Note that $C(\lambda)$ can be further minimised over $\lambda$ via numerical optim- 
isation $^{1}$.

Given $Y$, we can directly simulate $W$ via inverse transformation as the CDF of $\{W \mid Y\}$ is given as

$$
F(w \mid y)=\frac{1}{\left((1-y)^{-\alpha}-1\right)}\left[(1-y)^{-\alpha}-(1+w-y)^{-\alpha}\right], \quad 0<w<y
$$

which can be inverted explicitly and thus simulated via inverse transformation.

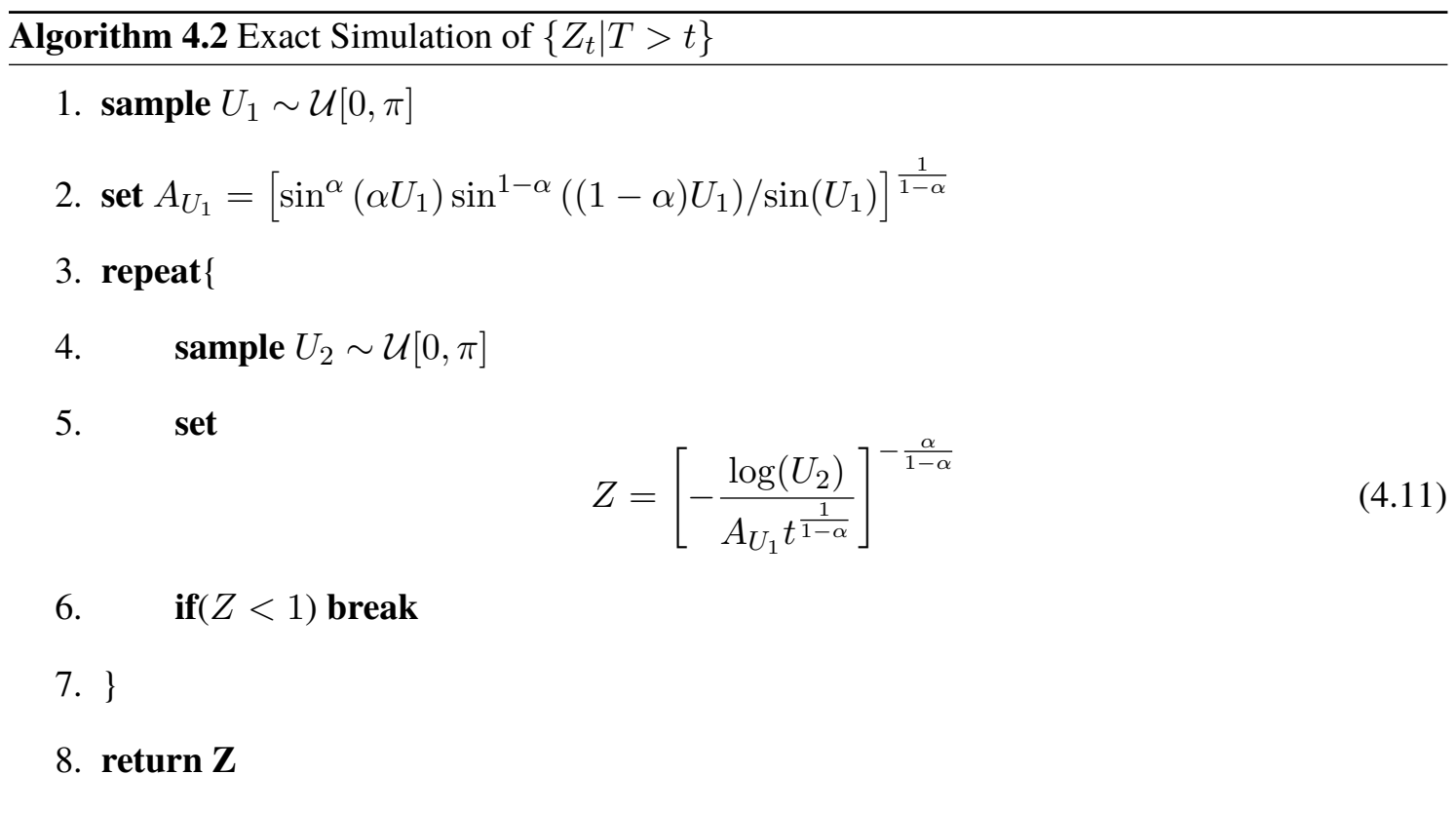

Proof. To generate $\left\{Z_{t} \mid T>t\right\}$, we can generate the stable process $S_{t}$ with the density

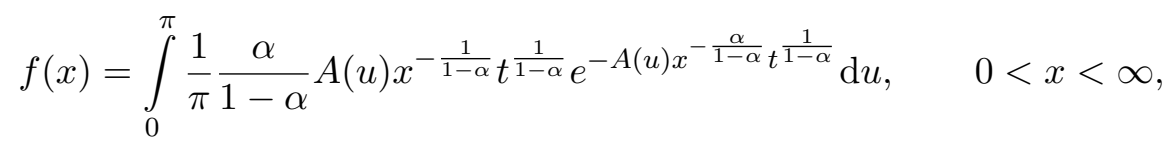

If $S_{t}<1$, then we accept the candidate. $S_{t}$ can be simulated via inverse transformation, the CDF is given as

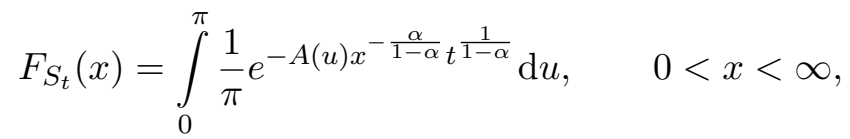

Hence, we first sample a uniform variable with domain $(0, \pi)$ and then sample $S_{t}$ via (4.11) through inverse transformation.

\footnotetext{
${ }^{1}$ The numerical optimisation in here and the other parts of this paper is only carried once before entering the loop. Hence, the optimisation will not slow down the entire simulation procedure.
} 
Hence, to generate a truncated stable sample $Z_{t}$, we implement Algorithm 4.3.

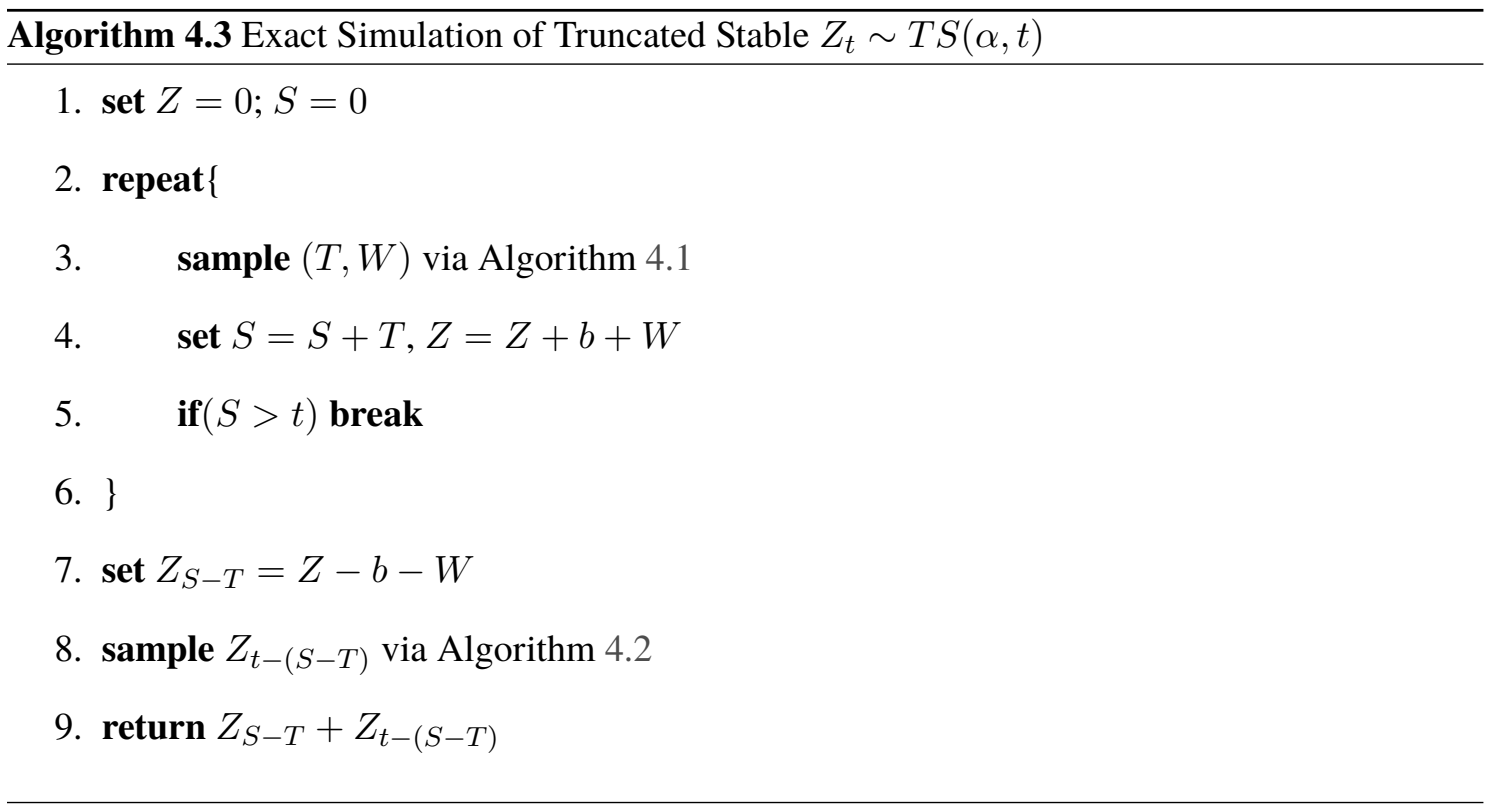

For $\mu>0$, the truncated tempered stable process can be simulated either via the marked renewal approach or via simple rejection method based on truncated stable process. Although we can use the marked renewal approach to generate this truncated tempered stable, due to the complexity of the analytical distribution of $(T, W)$, we therefore design an alternative simulation scheme based on the simulation algorithm for truncated stable process. As the density for the truncated stable is obtained by tempering exponential function to the truncated stable process, we can use an A/R scheme after generating the truncated stable sample. The procedures are illustrated in Algorithm 4.4.

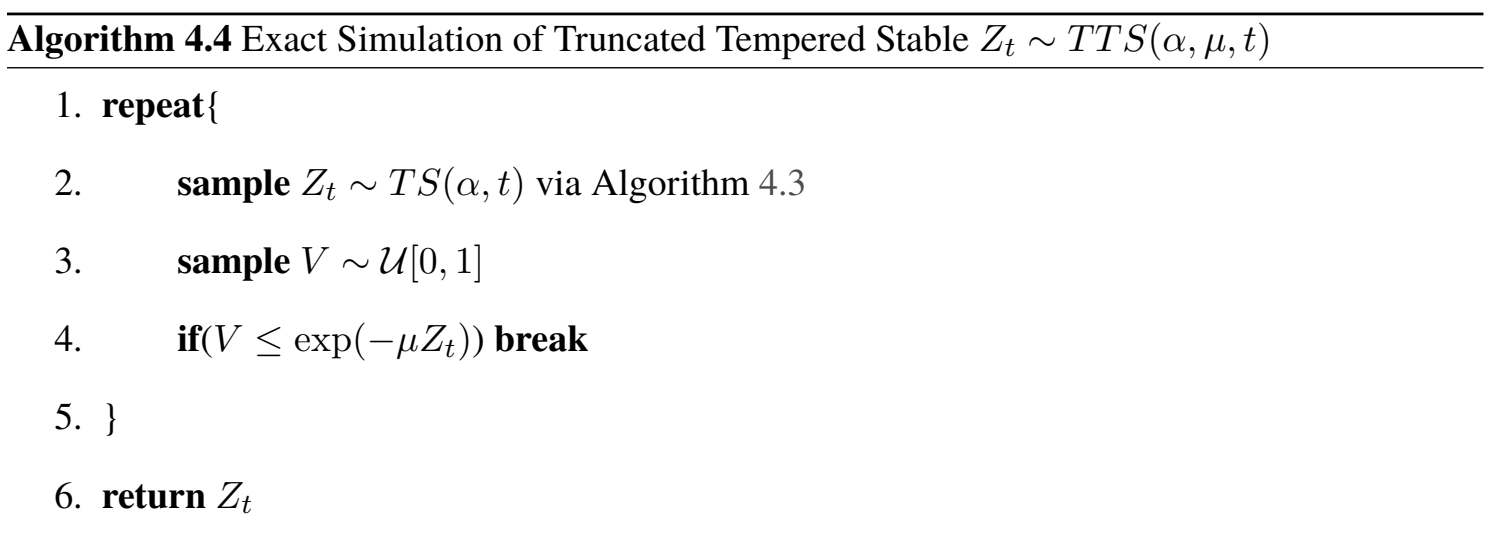

This algorithm relies on the fact that the density of the truncated tempered stable process is obtained by multiplying an exponential function to the density of the truncated stable process. The algorithm only works efficiently for smaller tilting parameter $\mu$. As the complexity of the algorithm is given by $\exp (\mu)$, the computation costs will increase rapidly when the tilting parameter increases. 
In order to improve the efficiency and reduce simulation time, we develop a decomposition scheme to reduce the size of the tilting parameter when $\mu$ is large, the details are provided in Theorem 4.1.

Theorem 4.1. The distribution of truncated tempered stable process $Z_{t} \sim T T S(\alpha, \mu, t)$ can be exactly decomposed as

$$
Z_{t} \stackrel{\mathcal{D}}{=} b \times \tilde{Z}_{t}+\sum_{i=1}^{N_{t}} J_{i}, \quad b \in(0,1)
$$

where

- $\tilde{Z}_{t} \sim T T S\left(\alpha, \mu b, t / b^{\alpha}\right)$;

- $N_{t}$ is a Poisson process with rate $\lambda t$, where ${ }^{2}$

$$
\lambda=\left[\left(b^{-\alpha} e^{-b \mu}-e^{-\mu}\right)+\mu^{\alpha}(\Gamma(1-\alpha, \mu)-\Gamma(1-\alpha, \mu b))\right] / \Gamma(1-\alpha) ;
$$

- $\left\{J_{i}\right\}_{i=1,2, \ldots}$ are i.i.d random variables with distribution

$$
f_{J_{i}}(z)=\frac{\alpha z^{-\alpha-1} e^{-\mu z}}{\left[\left(b^{-\alpha} e^{-b \mu}-e^{-\mu}\right)+\mu^{\alpha}(\Gamma(1-\alpha, \mu)-\Gamma(1-\alpha, \mu b))\right]}, \quad z \in(b, 1), \quad \forall i=1,2, \ldots
$$

Proof. For a truncated tempered stable process $Z_{t}$ with $\mu>0$, the Laplace transform is of the following form

$$
\begin{aligned}
\mathbb{E}\left[e^{-v Z_{t}}\right] & =\exp \left(-\frac{\alpha t}{\Gamma(1-\alpha)} \int_{0}^{1}\left(1-e^{-v z}\right) z^{-\alpha-1} e^{-\mu z} \mathrm{~d} z\right) \\
& =\exp \left(-\frac{\alpha t}{\Gamma(1-\alpha)} \int_{0}^{b}\left(1-e^{-v z}\right) \frac{e^{-\mu z}}{z^{\alpha+1}} \mathrm{~d} z\right) \exp \left(-\frac{\alpha t}{\Gamma(1-\alpha)} \int_{b}^{1}\left(1-e^{-v z}\right) \frac{e^{-\mu z}}{z^{\alpha+1}} \mathrm{~d} z\right) \\
& =\exp \left(-\frac{\alpha t}{b^{\alpha} \Gamma(1-\alpha)} \int_{0}^{1}\left(1-e^{-v b z}\right) \frac{e^{-\mu b z}}{z^{\alpha+1}} \mathrm{~d} z\right) \exp \left(-\frac{\alpha t}{\Gamma(1-\alpha)} \int_{b}^{1}\left(1-e^{-v z}\right) \frac{e^{-\mu z}}{z^{\alpha+1}} \mathrm{~d} z\right),
\end{aligned}
$$

where $b<1$. We can see that

1. The first part of (4.14) is the Laplace transform of $\tilde{Z}_{t} \sim T T S\left(\alpha, \mu b, t / b^{\alpha}\right)$ with Lévy measure

$$
\nu(\mathrm{d} z)=\frac{\alpha}{b^{\alpha} \Gamma(1-\alpha)} \frac{e^{-\mu b z}}{z^{\alpha+1}} \mathrm{~d} z
$$

2. The second part of (4.14) is the Laplace transform of a compound Poisson process. The

\footnotetext{
${ }^{2} \Gamma(\cdot, \cdot)$ is the upper incomplete gamma function.
} 
Laplace exponent can be rewritten as

$$
\lambda t \int_{0}^{\infty}\left(1-e^{-v z}\right) f_{J_{i}}(z) \mathrm{d} z,
$$

where $\lambda$ is of the form (4.12) and $f_{J_{i}}(\cdot)$ is of the form (4.13). As $\lambda<\infty$, this clearly indicates that (4.15) is the Laplace exponent of a compound Poisson process.

And the corresponding simulation algorithm based on the decomposition scheme is given as follows:

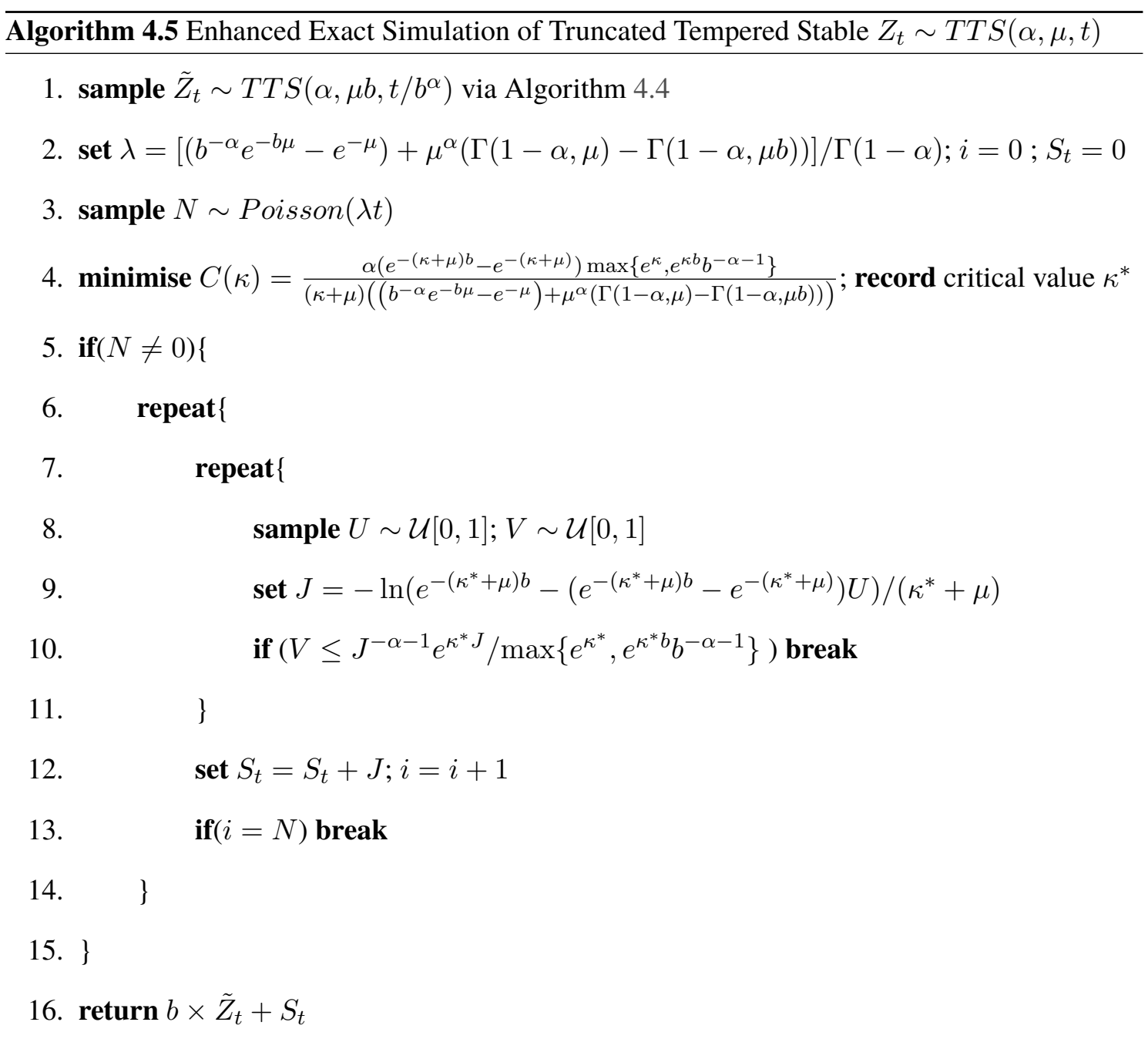

Proof. According to Theorem 4.1, $\tilde{Z}_{t} \sim T T S\left(\alpha, \mu b, t / b^{\alpha}\right)$ can be simulated via Algorithm 4.4, with tilting parameter $\mu b$. And the i.i.d jumps for the compound Poisson can be simulated via acceptance-rejection scheme, we choose an envelop random variable $J^{\prime}$ with density

$$
g(z)=\frac{(\kappa+\mu) e^{-(\kappa+\mu) z}}{e^{-(\kappa+\mu) b}-e^{-(\kappa+\mu)}}, \quad z \in(b, 1)
$$


the $\mathrm{CDF}$ is given by

$$
G(z)=\frac{e^{-(\kappa+\mu) b}-e^{-(\kappa+\mu) z}}{e^{-(\kappa+\mu) b}-e^{-(\kappa+\mu)}}, \quad z \in(b, 1)
$$

which can be inverted explicitly, i.e. $G^{-1}(u)=-\ln \left(e^{-(\kappa+\mu) b}-\left(e^{-(\kappa+\mu) b}-e^{-(\kappa+\mu)}\right) u\right) /(\kappa+\mu), u \in$ $(0,1)$, and the random variable $J^{\prime}$ can be simulated via inverse transformation by setting $J^{\prime}=$ $-\ln \left(e^{-(\kappa+\mu) b}-\left(e^{-(\kappa+\mu) b}-e^{-(\kappa+\mu)}\right) U\right) /(\kappa+\mu)$ with $U \sim \mathcal{U}[0,1]$. The A/R constant is given as

$$
C(\kappa)=\frac{\alpha\left(e^{-(\kappa+\mu) b}-e^{-(\kappa+\mu)}\right) \max \left\{e^{\kappa}, \frac{e^{\kappa b}}{b^{\alpha+1}}\right\}}{(\kappa+\mu)\left[\left(b^{-\alpha} e^{-b \mu}-e^{-\mu}\right)+\mu^{\alpha}(\Gamma(1-\alpha, \mu)-\Gamma(1-\alpha, \mu b))\right]},
$$

where $\kappa^{*}$ is the critical value that minimises $C(\kappa)$.

Since we break the truncated tempered stable process into a truncated tempered process with a smaller tilting parameter and a compound Poisson process, the computation costs will be reduced for the truncated tempered stable process with smaller tilting parameter, but there will be extra costs to generate the compound Poisson process. Therefore, we also need to consider the efficiency to generate the compound Poisson process to choose an optimal $b$ to decompose the process.

Finally, for $\mu<0, Z_{t}$ is a well-defined Lévy subordinator. Based on distributional decomposition, this process can be split into a truncated stable process, and a compound Poisson process. Details of the decomposition scheme are given in Theorem 4.2.

Theorem 4.2. Let $Z_{t}$ be a Lévy subordinator with Lévy measure

$$
\nu(\mathrm{d} z)=\frac{\alpha z^{-\alpha-1} e^{|\mu| z}}{\Gamma(1-\alpha)} \mathbf{1}_{\{0<z<1\}}, \quad \alpha \in(0,1), \quad \mu<0
$$

we have

$$
Z_{t} \stackrel{\mathcal{D}}{=} \bar{Z}_{t}+\sum_{i=1}^{N_{t}} Y_{i}
$$

where

- $\bar{Z}_{t} \sim T S(\alpha, t)$

- $N_{t}$ is a Poisson process with rate $\varrho t$, where $\varrho=\frac{\alpha}{\Gamma(1-\alpha)} \int_{0}^{1}\left(e^{|\mu| z}-1\right) z^{-\alpha-1} \mathrm{~d} z$.

- $\left\{Y_{i}\right\}_{i=1,2, \ldots}$ are i.i.d random variables with distribution

$$
f_{Y_{i}}(z)=\frac{1}{F} \frac{e^{|\mu| z}-1}{z^{\alpha+1}}, \quad z \in(0,1)
$$

with $F=\int_{0}^{1}\left(e^{|\mu| z}-1\right) z^{-\alpha-1} \mathrm{~d} z<\infty$. 
Proof. The Laplace transform of $Z_{t}$ can be expressed as follows,

$\mathbb{E}\left[e^{-v Z_{t}}\right]=\exp \left(-\frac{\alpha t}{\Gamma(1-\alpha)} \int_{0}^{1}\left(1-e^{-v z}\right) \frac{1}{z^{\alpha+1}} \mathrm{~d} z\right) \exp \left(-\frac{\alpha t F}{\Gamma(1-\alpha)} \int_{0}^{1}\left(1-e^{-v z}\right) \frac{e^{|\mu| z}-1}{F z^{\alpha+1}} \mathrm{~d} z\right)$,

where $F=\int_{0}^{1}\left(e^{|\mu| z}-1\right) z^{-\alpha-1} \mathrm{~d} z$ is a finite constant. We can see that the first part of (4.17) ia a truncated stable process with stability $\alpha$. The second part of (4.17) is a compound Poisson process with Poisson rate $\frac{\alpha t F}{\Gamma(1-\alpha)}$ and the density of the i.i.d jumps, namely $f_{Y_{i}}(i)$, of the form in (4.16) is a well-defined density as $f_{Y_{i}}(z)>0$ for all $z \in(0,1)$ and $\int_{0}^{\infty} f_{Y_{i}}(z) \mathrm{d} z=1$ as $F$ is finite.

Based on Algorithm 4.3 and Theorem 4.2, to generate $Z_{t}$ with $\mu<0$, one could follow Algorithm 4.6.

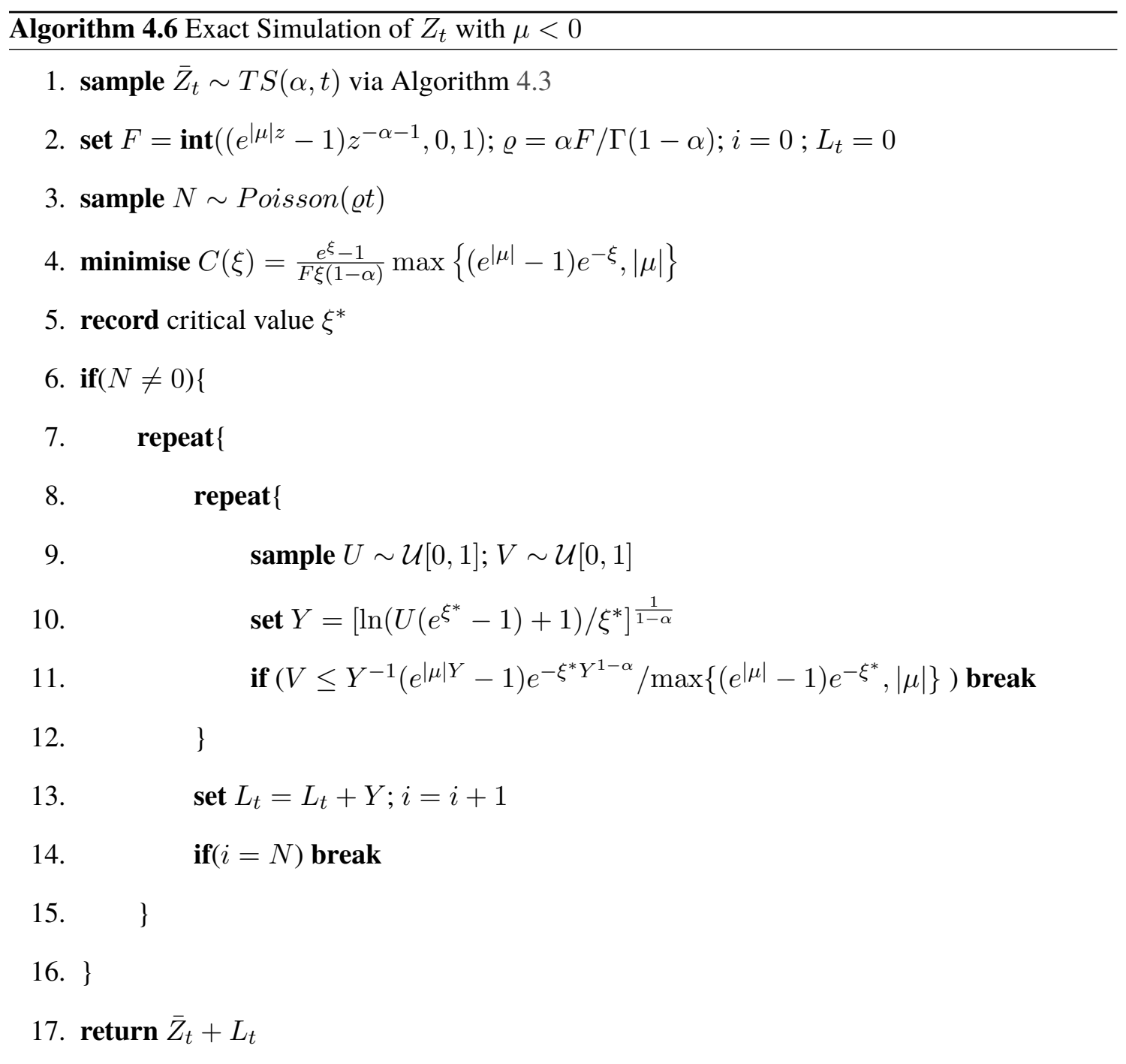


Proof. According to Theorem 4.2, $Z_{t}$ with $\mu<0$ can be decomposed into a truncated stable process $\bar{Z}_{t}$ with stability index $\alpha$ and a compound Poisson process $\sum_{i=1}^{N_{t}} Y_{i} . \bar{Z}_{t}$ can be directly simulated via Algorithm 4.3. The i.i.d jumps of the compound Poisson can be simulated via an acceptancerejection scheme. We choose an envelope random variable $Y^{\prime}$ with density

$$
g(z)=\frac{\xi(1-\alpha)}{e^{\xi}-1} \frac{e^{\xi z^{1-\alpha}}}{z^{\alpha}}, \quad 0<z<1
$$

the $\mathrm{CDF}$ is given by

$$
G(z)=\frac{e^{\xi z^{1-\alpha}}-1}{e^{\xi}-1}, \quad 0<z<1
$$

which can be inverted explicitly, i.e.

$$
G^{-1}(u)=\left[\ln \left(u\left(e^{\xi}-1\right)+1\right) / \xi\right]^{\frac{1}{1-\alpha}}, \quad 0<u<1 .
$$

and the random variable can be simulated via inverse transformation by setting $Y^{\prime}=\left[\ln \left(U\left(e^{\xi}-\right.\right.\right.$ $1)+1) / \xi]^{\frac{1}{1-\alpha}}$ with $U \sim \mathcal{U}[0,1]$. To find the A/R constant, we have

$$
\begin{aligned}
\frac{f_{Y_{i}}(z)}{\left.g_{(} z\right)} & =\frac{e^{\xi}-1}{F \xi(1-\alpha)} \frac{\left(e^{|\mu| z}-1\right) e^{-\xi z^{1-\alpha}}}{z} \\
& \leq \frac{e^{\xi}-1}{F \xi(1-\alpha)} \max \left\{\left(e^{|\mu|}-1\right) e^{-\xi},|\mu|\right\}
\end{aligned}
$$

The $\mathrm{A} / \mathrm{R}$ constant depends on the input $\mu$, in order to obtain high acceptance rate, we use numerical optimization to find the optimal $\xi^{*}$ that minimises (4.18).

\subsection{Numerical Studies}

In this section, we illustrate the performance and effectiveness of our exact simulation schemes through extensive numerical experiments. The simulation experiments are all conducted on a normal laptop with Intel Core i7-6500U CPU@2.50GHz processor, 8.00GB RAM,Windows 10 Home and 64-bit Operating System. The algorithms are coded and performed in R.3.6.0, and computing time is measured by the elapsed CPU time in seconds. Numerical validation and test for our algorithms are based on the PDF and CDF of truncated stable process and truncated tempered stable process, which can be calculated by inverting the Laplace transform numerically ${ }^{3}$. In particular, we assess the goodness-of-fit by comparing the CDFs and PDFs obtained by Algorithm 4.3, 4.5 and 4.6 and by the numerical inverse of Laplace transform. The associated plots of CDFs, PDFs

\footnotetext{
${ }^{3}$ A variety of methods are available for numerically inverting Laplace transforms with high accuracy, such as Gaver (1966), Stehfest (1970), Abate and Whitt (1992, 1995, 2006). Here, we use the Euler scheme in Abate and Whitt (2006).
} 

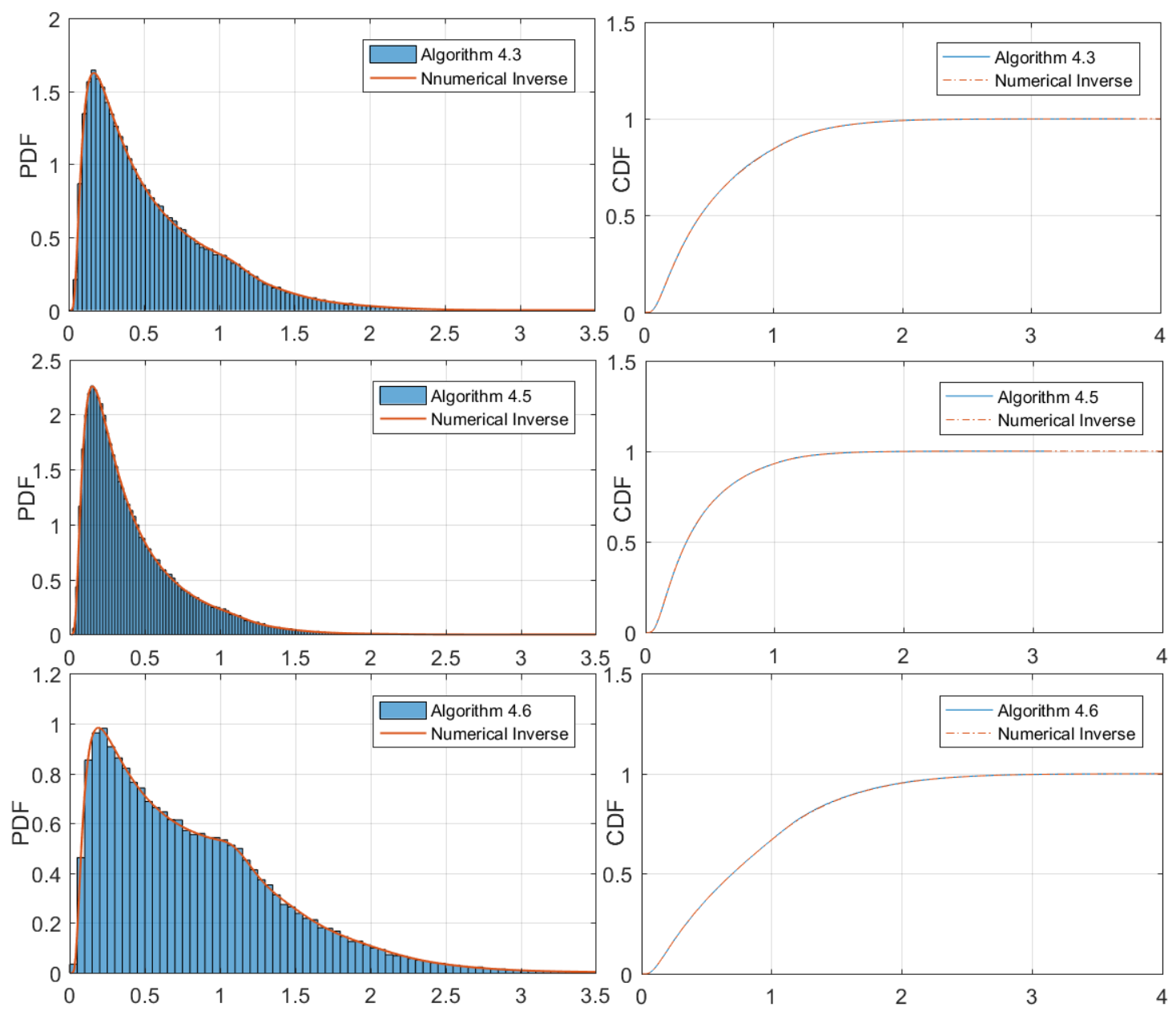

Figure 3: Comparison of the empirical CDF/PDF for Algorithm 4.3, 4.5 and 4.6 with the CDF/PDF obtained via numerical inverse under the parameter setting $t=1, \alpha=0.5$ for Algorithm $4.3, t=1, \alpha=$ $0.5, \mu=1$ for Algorithm 4.5, and $t=1, \alpha=0.5, \mu=-1$ for Algorithm 4.6.

and histograms are illustrated in Figure 3. Overall, we can observe that each of these algorithms can achieve a very high level of accuracy, and the simulated CDF and PDF are fitted well to the associated numerical inversion.

For truncated stable process, we establish a comparison of CPU time for Algorithm 4.3 with stability index $\alpha=0.1,0.2,0.3, \ldots, 0.9$. The numerical results in detail is reported in Table 1 . We can see that Algorithm 4.3 is more efficient for large stability parameter $\alpha$. This is due to the fact that the $\mathrm{A} / \mathrm{R}$ constant $C(\lambda)$ in (4.10) is smaller for large $\alpha$, i.e. the acceptance rate to sample $(T, W)$ using Algorithm 4.1, Figure 4, is higher for large $\alpha$. Larger $t$ also requires more computation time as one has to generate more hitting times to break the marked renewal routine. We also implement a comparison between Algorithm 4.3 against the algorithm suggested in Chi (2012). We compared the computation time to generate 100, 000 samples using these two algorithms for different values of $\alpha$. We can see that sampling based on Algorithm 4.3 is much faster than algorithm in Chi (2012). 


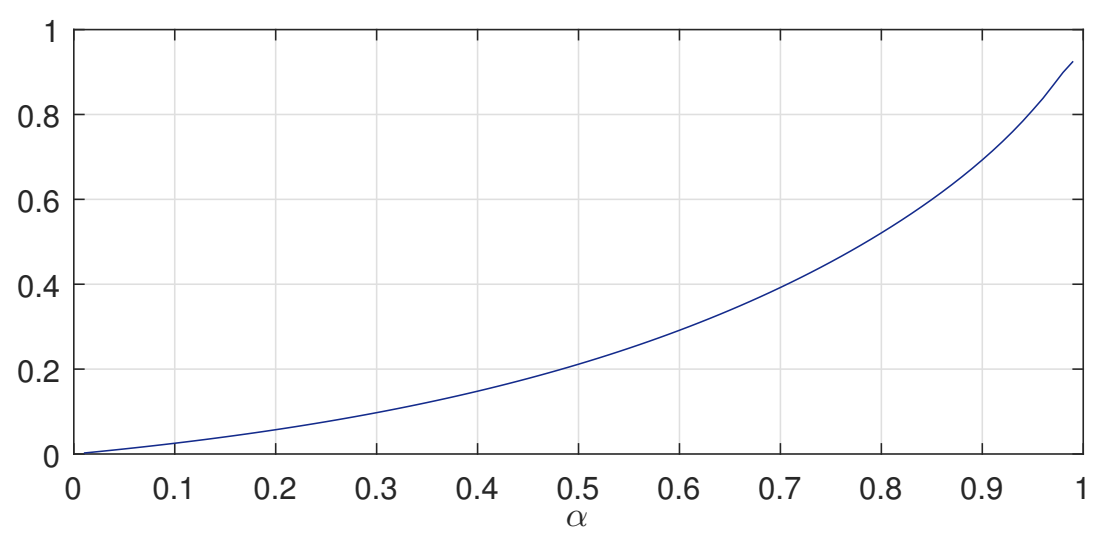

Figure 4: Acceptance rate for Algorithm 4.1 to sample $(T, W)$ for $\alpha \in(0,1)$.

Table 1: Comparison of the CPU times for Algorithm 4.3 and Algorithm suggested in Chi (2012) for 100, 000 replications, based on parameter setting $\alpha=0.1,0.2,0.3,0.4,0.5,0.6,0.7,0.8,0.9$ and $t=1$, respectively.

\begin{tabular}{c|r|r|r|r|r|r|r|r|r}
\hline \hline$\alpha$ & 0.10 & 0.20 & 0.30 & 0.40 & 0.50 & 0.60 & 0.70 & 0.80 & 0.90 \\
\hline Algorithm 4.3 & 9.38 & 5.47 & 4.89 & 3.57 & 2.40 & 2.75 & 2.56 & 2.36 & 2.19 \\
\hline Chi (2012) & 480.37 & 495.21 & 553.78 & 538.80 & 557.83 & 581.21 & 613.72 & 639.30 & 682.49 \\
\hline \hline
\end{tabular}

For instance, for $\alpha=0.5$ and $t=1$, Algorithm 4.3 is 230 times faster than Chi (2012) for 100, 000 replications. The out-performance of our algorithm would even become much more substantial when $t$ increases.

For the truncated tempered stable process, we carry out a comparison between Algorithm 4.4 and 4.5. The results are reported in Table 2. We can see that when $\mu$ and $t$ are small, the time needed for two algorithms are more or less the same. However, when $\mu$ and $t$ are larger, Algorithm 4.5 outperforms Algorithm 4.4 in terms of computation time.

\subsection{Truncated Stable Process and Perpetuities with Hyperbolic Discounting}

The truncated stable process is also closely related with the present value of hyperbolic discounting perpetuity $X$ of the form

$$
X=\sum_{i=1}^{\infty} h\left(T_{i}\right)
$$

where $T_{i}$ is the time of the $i^{t h}$ event of a Poisson process $N_{t}$ with rate $\lambda$ and $h(\cdot)$ is the discounting function of the form

$$
h(t)=(1+k t)^{-\gamma}, \quad k \in \mathbb{R}^{+}, \gamma \in(1, \infty) .
$$

The Laplace transform of $X$ is provided in Theorem 4.3.

Theorem 4.3. Consider the perpetuity $X$ with hyperbolic discounting in (4.19), the Laplace trans- 
Table 2: Comparison between the true means and the associated simulation results based on parameter setting $t=0.5,4, \alpha=0.5, \mu=1$ for Algorithm 4.4, 4.5.

\begin{tabular}{c|cccccc|cccccc}
\hline \hline Paths & 1,000 & 4,000 & 16,000 & 64,000 & 256,000 & $1,024,000$ & 1,000 & 4,000 & 16,000 & 64,000 & 256,000 & $1,024,000$ \\
\hline \hline & \multicolumn{1}{c|}{$\alpha=0.5$} & $t=0.5$ & $\mu=1$ & & & & $\alpha=0.5$ & $t=4$ & $\mu=1$ & \\
\hline Algorithm 4.4 & 0.15 & 0.56 & 2.08 & 7.47 & 28.41 & 117.74 & 0.14 & 0.61 & 2.73 & 10.67 & 43.81 & 167.59 \\
\hline Algorithm 4.5 & 0.11 & 0.35 & 1.23 & 4.14 & 17.31 & 76.69 & 0.12 & 0.40 & 1.65 & 5.45 & 23.41 & 90.74 \\
\hline \hline
\end{tabular}

form is given as follows,

$$
\mathbb{E}\left[e^{-v X}\right]=\exp \left(-\frac{\lambda}{k \gamma} \int_{0}^{1}\left(1-e^{-v u}\right) u^{-1-\frac{1}{\gamma}} \mathrm{d} u\right), \quad v \in \mathbb{R}^{+}
$$

Proof. The infinitesimal generator $\mathcal{A}$ of the process $(X, t)$ acting on any function $f(x, t)$ within its domain $\Omega(\mathcal{A})$ is given by

$$
\mathcal{A} f(x, t)=\frac{\partial f}{\partial t}+\lambda(f(x+h(t))-f(x))
$$

By applying the piecewise-deterministic Markov processes theory and martingale approach, we can derive the conditional Laplace transform of $X$. More precisely, set $\mathcal{A} f(x, t)=0$, we try a solution of the form $e^{-v x} g(t)$ with $v \in \mathbb{R}^{+}$, where $g(t)$ is deterministic and differentiable function of $t$. Then, we get

$$
g^{\prime}(t)+\lambda g(t)\left(e^{-v h(t)}-1\right)=0,
$$

Hence, the martingale is of the form

$$
e^{-v X} \exp \left(\lambda \int_{0}^{t}\left(1-e^{-v h(s)}\right) \mathrm{d} s\right)
$$

By martingale property, we obtain

$$
\begin{aligned}
\mathbb{E}\left[e^{-v X}\right] & =\exp \left(-\lambda \int_{0}^{\infty}\left(1-e^{-v h(s)}\right) \mathrm{d} s\right) \\
& =\exp \left(-\frac{\lambda}{k \gamma} \int_{0}^{1}\left(1-e^{-v u}\right) u^{-1-\frac{1}{\gamma}} \mathrm{d} u\right) .
\end{aligned}
$$

We have $X \stackrel{\mathcal{D}}{=} Z_{t}$, where $Z_{t}$ represents the position of a truncated stable process at time $t=$ $\lambda \Gamma(1-\alpha) /[\alpha k \gamma]$, such that $Z_{t} \sim T S(1 / \gamma, \lambda \Gamma(1-\alpha) /[\alpha k \gamma])$. Therefore, we can use Algorithm 4.3 to simulate the hyperbolic discounting perpetuity $X$. Figure 5 shows the histograms of the hyperbolic discounting perpetuity $X$ with different parameters of $\gamma$. 

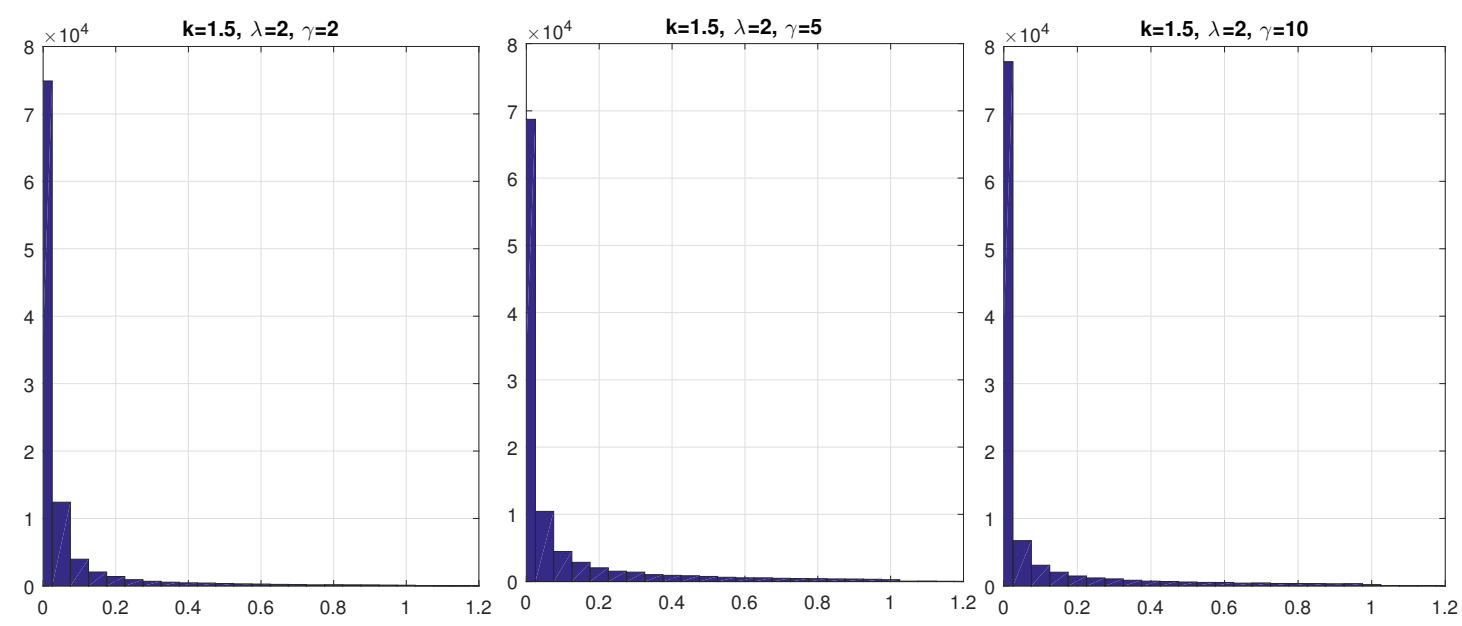

Figure 5: Histograms of Hyperbolic Discounting Perpetuity $X$ under the parameter setting $\gamma=2,5,10$, $k=1.5, \lambda=2$, respectively.

For a more general hyperbolic discounting perpetuity $X$ of the form

$$
X=\sum_{i=1}^{\infty} Y_{i} h\left(T_{i}\right)
$$

with $\left\{Y_{i}\right\}_{i=1,2, \ldots}$ being i.i.d random variables with finite mean, the value of $X$ turns out to be the position of a truncated Lévy subordinator $X_{t}$ at the fixed time $t=\frac{\lambda}{\kappa \gamma}$ with Lévy measure

$$
\nu(\mathrm{d} x)=t x^{-1-\frac{1}{\gamma}} \int_{x}^{\infty} y^{\frac{1}{\gamma}} f_{Y}(y) \mathrm{d} y,
$$

where $f_{Y}(\cdot)$ is the density of $Y_{i}$. We could apply the decomposition scheme suggested in Dassios et al. (2019) to simulate the general hyperbolic discounting perpetuity.

\section{Truncated Lévy Related Processes}

\subsection{Two-sided symmetric Lévy Process}

Based on the marked renewal scheme for truncated Lévy process, we can also simulate two-sided symmetric truncated Lévy processes. Since Lévy process with bounded variation can be represented as the difference of two independent subordinators, for two-sided symmetric truncated Lévy process $\hat{Z}_{t}$ with characteristic function

$$
\mathbb{E}\left[e^{\mathrm{i} \theta \hat{Z}_{t}}\right]=\exp \left(t \int_{-b}^{b}\left(e^{\mathrm{i} \theta z}-1\right) \nu(\mathrm{d} z)\right), \quad \theta \in \mathbb{R}
$$




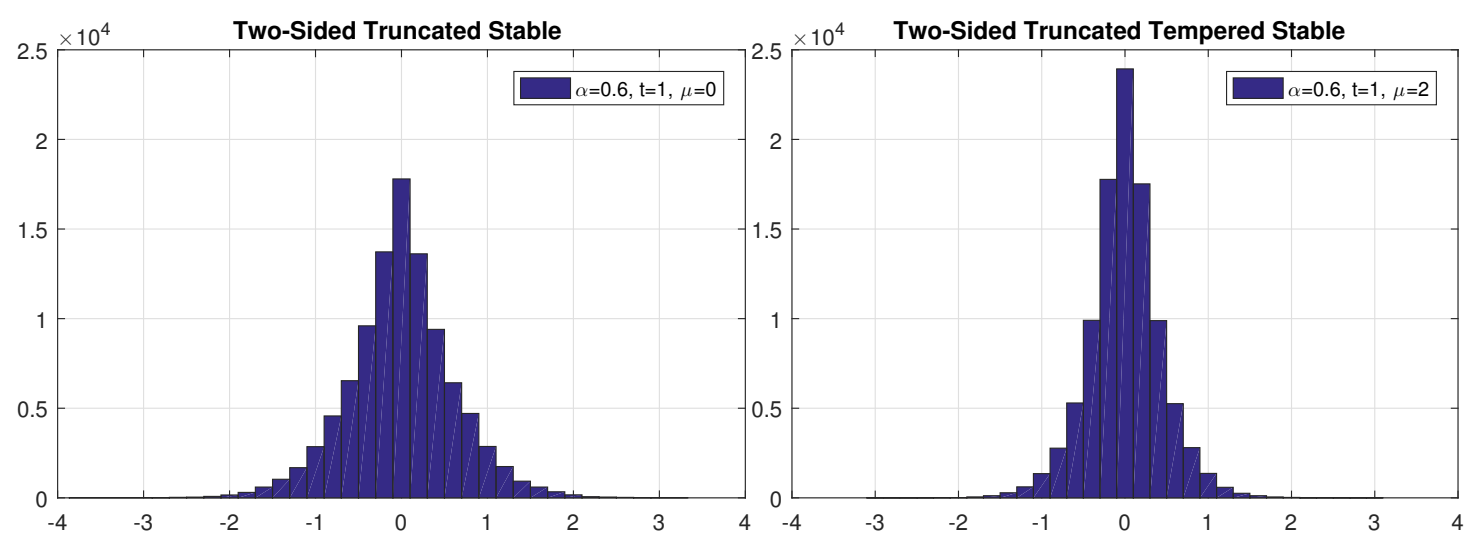

Figure 6: Histograms of two-sided truncated stable and truncated tempered stable, $\hat{Z}_{t}$, under the parameter setting $\alpha=0.6, t=1, b=1$, and $\mu=0,2$, respectively.

where $\nu$ is the Lévy measure on $R$ that satisfies the following conditions

$$
\int_{\mathbb{R}} \min \{1, z\} \nu(\mathrm{d} z)<\infty
$$

and $\nu(z)=\nu(-z), \quad \forall z \in(-b, b)$.

Proposition 5.1. The law of $\hat{Z}_{t}$ with characteristic function (5.1) can be exactly decomposed by

$$
\hat{Z}_{t} \stackrel{\mathcal{D}}{=} Z_{t}^{+}-Z_{t}^{-}
$$

where $Z_{t}^{+}, Z_{t}^{-}$are independent truncated Lévy subordinators.

Proof. The characteristic function of $\hat{Z}_{t}$ can be expressed as

$$
\begin{aligned}
\mathbb{E}\left[e^{\mathrm{i} \theta \hat{Z}_{t}}\right] & =\exp \left(t \int_{-b}^{b}\left(e^{\mathrm{i} \theta z}-1\right) \nu(|z|) \mathrm{d} z\right) \\
& =\exp \left(t \int_{0}^{b}\left(e^{\mathrm{i} \theta z}-1\right) \nu(|z|) \mathrm{d} z\right) \exp \left(t \int_{0}^{b}\left(e^{-\mathrm{i} \theta z}-1\right) \nu(|z|) \mathrm{d} z\right) \\
& =\mathbb{E}\left[e^{\mathrm{i} \theta\left(Z_{t}^{+}-Z_{t}^{-}\right)}\right]
\end{aligned}
$$

where $Z_{t}^{+}, Z_{t}^{-}$are independent truncated Lévy subordinators with Lévy measure $\nu(z)$ on $z \in$ $(0, b)$.

In Figure 6, we illustrate the histograms of the two-sided truncated stable and truncated tempered stable under different parameter settings. Except the two-sided symmetric truncated Lévy process, a new class of two-sided symmetric Lévy processes, namely Brownian motions subordinated by truncated Lévy subordinators, can also be simulated based on the marked renewal simulation 

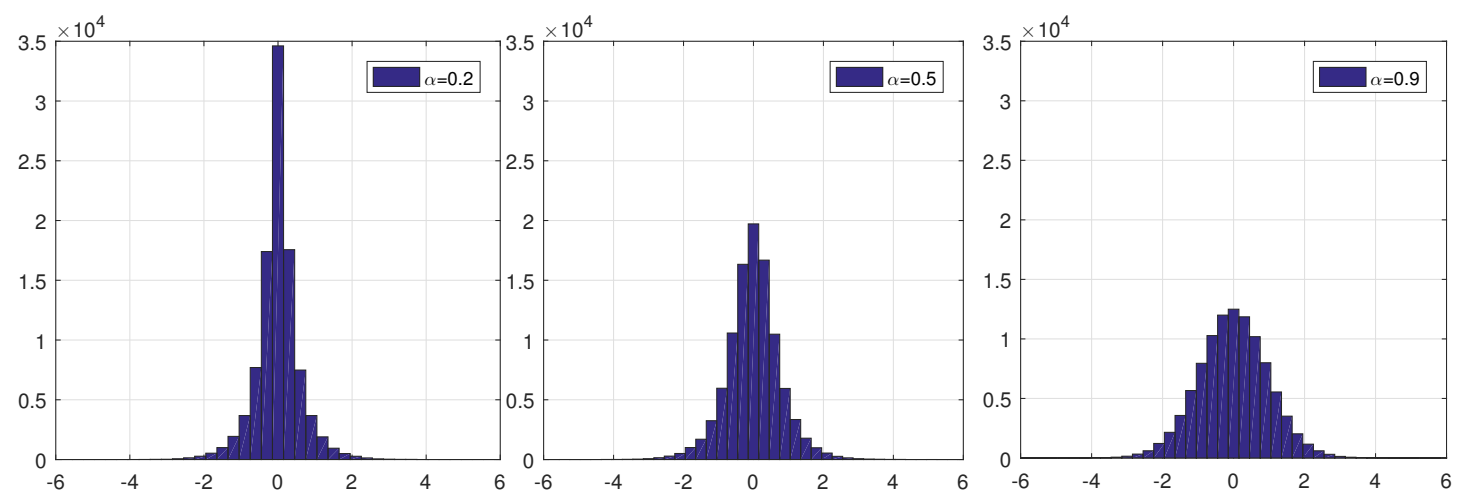

Figure 7: Histograms of Brownian motion subordinated by truncated stable process $B_{Z_{t}}$ under the parameter setting $t=1, b=1$, and $\alpha=0.2,0.5,0.9$, respectively.

scheme. Let $B_{t}$ be a standard Brownian motion, $Z_{t}$ be an independent truncated Lévy subordinator with Lévy measure $\nu_{Z}$ on $(0, b)$. The process $B_{Z_{t}}$ is a two-sided symmetric Lévy process, the associated Lévy measure is of the following form,

$$
\nu(\mathrm{d} x)=\int_{0}^{b} \frac{e^{-\frac{x^{2}}{2 z}}}{\sqrt{2 \pi z}} \nu_{Z}(\mathrm{~d} z) \mathrm{d} x, \quad x \in \mathbb{R} .
$$

Example 5.1. Let $Z_{t}$ be a truncated stable process with truncation level $b, B_{Z_{t}}$ is a two-sided symmetric Lévy process with Lévy measure

$$
\nu(\mathrm{d} x)=\frac{t}{2^{1-\alpha} \pi} \frac{\Gamma\left(\alpha+1 / 2, x^{2} /[2 b]\right)}{x^{1+2 \alpha}} \mathrm{d} x, \quad x \in \mathbb{R} .
$$

When $\alpha=1 / 2, B_{Z_{t}}$ is a rapidly decaying tempered stable with stability 1 , see Kim et al. (2010), the Lévy measure is given as

$$
\nu(\mathrm{d} x)=\frac{t}{\sqrt{2} \pi} \frac{e^{-\frac{x^{2}}{2 b}}}{x^{2}} \mathrm{~d} x, \quad x \in \mathbb{R} .
$$

In Figure 7, we show the histograms for $B_{Z_{t}}$ under $t=1$ and $\alpha=0.2,0.5,0.9$.

\subsection{Truncated Lévy Driven Ornstein-Uhlenbeck Process}

A Lévy driven Ornstein-Uhlenbeck process is the analogue of an ordinary Gaussian OU process with its Brownian motion part replaced by a Lévy process, Uhlenbeck and Ornstein (1930). The process is defined via stochastic differential equation as below,

Definition 5.1 (Lévy Driven Ornstein-Uhlenbeck Process). $X_{t}$ is a Lévy driven Ornstein-Uhlenbeck 
process that satisfies the stochastic differential equation (SDE)

$$
\mathrm{d} X_{t}=-\delta X_{t} \mathrm{~d} t+\varrho \mathrm{d} Z_{t}, \quad t \geq 0,
$$

where

- $\varrho>0$ is a positive constant;

- $\delta>0$ is the constant rate of exponential decay;

- $Z_{t}$ is a Lévy subordinator, which is called the background driving Lévy process (BDLP) of a non-Gaussian OU process.

This class of stochastic processes has been extensively studied in the literature, see Wolfe (1982), Sato and Yamazato (1984), Barndorff-Nielsen (1998) and Barndorff-Nielsen et al. (1998). Nowadays, these processes have been widely used as the continuous-time stochastic models for the observed behaviour of price dynamics in finance and economics, see Barndorff-Nielsen and Shephard (2001b, 2002). It has also been used in option pricing, see Nicolato and Venardos (2003), Kallsen et al. (2011) and Li and Linetsky (2014), and for describing high-frequency financial data in market microstructure, see Barndorff-Nielsen and Shephard (2003a,b) and Todorov and Tauchen (2006).

Due to numerous applications of these models, the availability of efficient and accurate simulation algorithms is particularly important in the context of model validation and statistical inference, as well as for risk analysis and derivative pricing. The simulation design for exactly sampling without bias has been recently brought to the attention in the literature. Zhang and Zhang (2008); Zhang (2011); Qu et al. (2019); Dassios et al. (2017b) suggested exact simulation algorithms for several types of Lévy driven OU processes with BDLP being Gamma processes, inverse Gaussian processes, and tempered stable processes, etc. For the truncated Lévy driven OU processes, based on the decomposition scheme developed in Qu et al. (2019); Dassios et al. (2017b); Qu (2019) and the marked renewal simulation scheme for the truncated Lévy subordinator, one could also exact simulate truncated Lévy driven OU processes. In the rest of this section, we consider a typical example, i.e. the truncated stable driven OU process to demonstrate the methodology.

\section{Exact Simulation of truncated stable driven OU process}

Let $X_{t}$ be an OU process following the SDE in (5.4), with $Z_{t}$ being a truncated stable process with truncation level $b$. Given $\tau \in \mathbb{R}^{+}$, the distribution of $X_{t+\tau}$ conditional on $X_{t}$ can be decomposed 
into three basic components: one deterministic trend, one truncated stable, one compound Poisson. The details are provided in Proposition 5.2.

Proposition 5.2. The distribution of $X_{t+\tau}$ conditional on $X_{t}$ can be exactly decomposed as

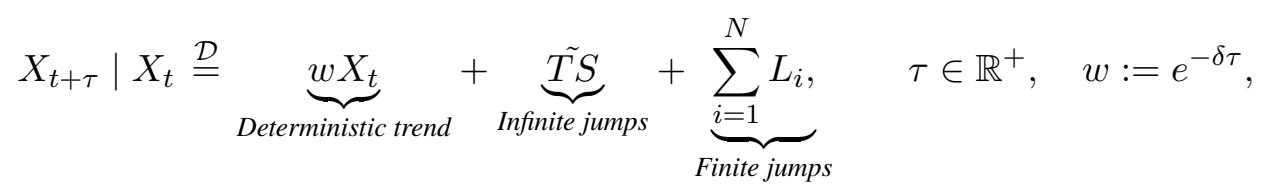

where

- TS is a truncated stable with Lévy measure

$$
\nu(\mathrm{d} x)=\frac{\left(1-w^{\alpha}\right) \varrho}{\delta \Gamma(1-\alpha)} \frac{1}{x^{\alpha+1}} \mathbf{1}_{\{0<x<b w\}} \mathrm{d} x .
$$

- $N$ is a Poisson random variable of rate $\frac{\varrho \zeta}{\delta \Gamma(1-\alpha)}$, where

$$
\zeta=\frac{b^{-\alpha}}{\alpha}\left[\alpha \log (w)+w^{-\alpha}-1\right]
$$

- $\left\{L_{i}\right\}_{i=1,2, \ldots}$ are i.i.d random variables with density

$$
f_{L_{i}}(x)=\frac{x^{-\alpha}-b^{-\alpha}}{\zeta x}, \quad x \in(b w, b),
$$

which can be simulated via the following $A / R$ procedures:

(1) sample $U \sim \mathcal{U}(0,1)$; set $X=b\left[w^{-\alpha}-\left(w^{-\alpha}-1\right) U\right]^{-\frac{1}{\alpha}}$;

(2) sample $V \sim \mathcal{U}(0,1)$, if

$$
V \leq\left(1-b^{-\alpha} X^{\alpha}\right) /\left(1-w^{\alpha}\right)
$$

then, accept X; Otherwise, reject this candidate and go back to Step (1).

Proof. According to Qu (2019), given the Lévy measure $Z_{t}$ in (4.1) with $\mu=0$, the Laplace transform of $X_{t+\tau}$ given $X_{t}$ can be expressed as

$$
\begin{aligned}
& \mathbb{E}\left[e^{-v X_{t+\tau}} \mid X_{t}\right] \\
= & e^{-v w X_{t}} \exp \left(-\frac{\alpha \varrho}{\delta \Gamma(1-\alpha)} \int_{v w}^{v} \frac{1}{u} \int_{0}^{b}\left(1-e^{-u y}\right) y^{-1-\alpha} \mathrm{d} y \mathrm{~d} u\right) \\
= & e^{-v w X_{t}} \exp \left(-\frac{\alpha \varrho}{\delta \Gamma(1-\alpha)} \int_{0}^{b}\left(1-e^{-v x}\right) \frac{1}{x} \int_{x}^{\frac{x}{w} \wedge b} y^{-1-\alpha} \mathrm{d} y \mathrm{~d} x\right)
\end{aligned}
$$



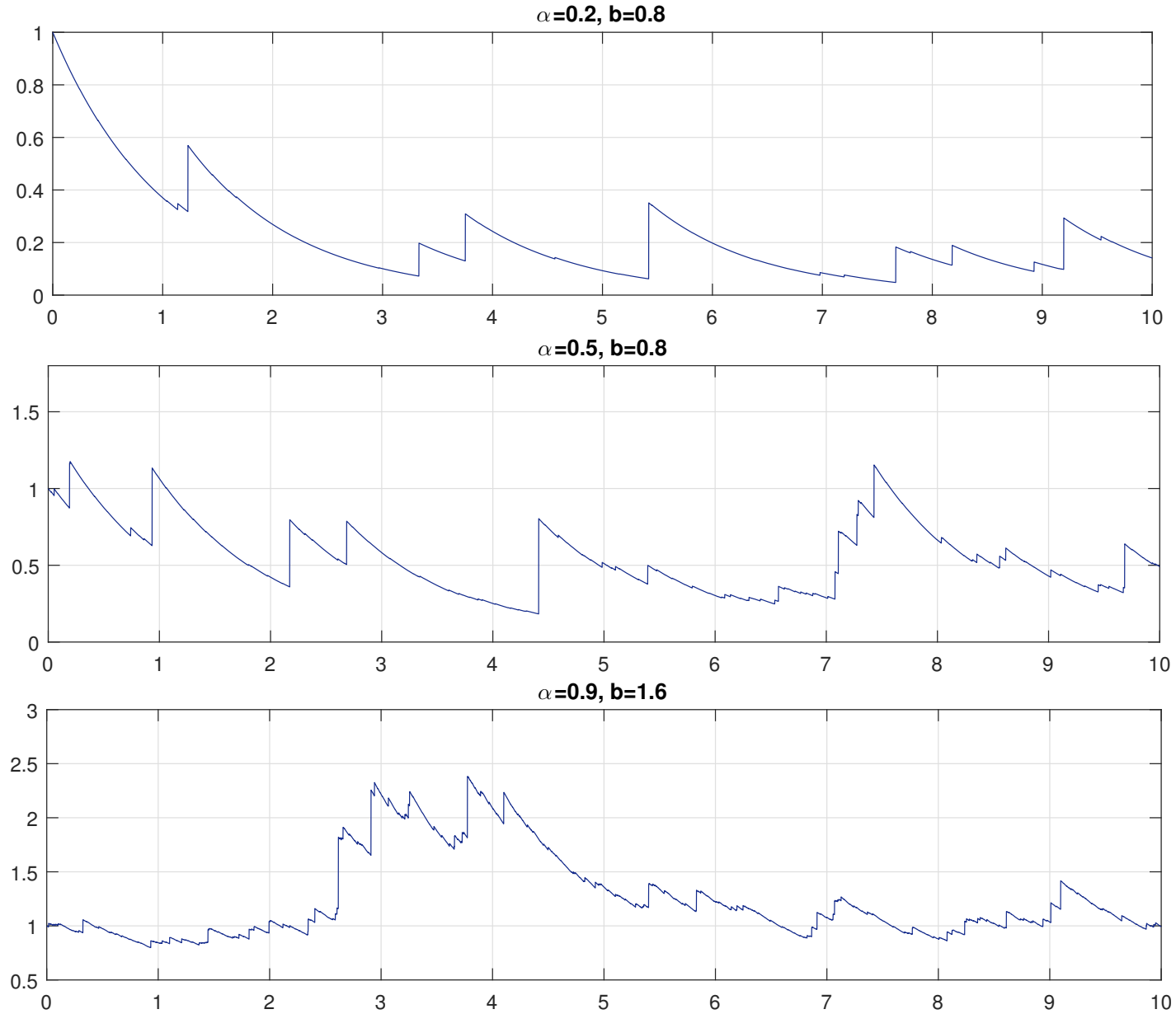

Figure 8: Simulated pathes of truncated stable driven OU processes, with the parameter setting $\left(\delta, \varrho, X_{0}\right)=$ $(1,1,1)$ within the time period of $[0,10]$ and 10,000 equally-spaced discretisation steps.

$$
=e^{-v w X_{t}} \exp \left(-\frac{\left(1-w^{\alpha}\right) \varrho}{\delta \Gamma(1-\alpha)} \int_{0}^{b w}\left(1-e^{-v x}\right) \frac{1}{x^{1+\alpha}} \mathrm{d} x\right) \exp \left(-\frac{\varrho \zeta}{\delta \Gamma(1-\alpha)} \int_{b w}^{b}\left(1-e^{-v x}\right) \frac{x^{-\alpha}-b^{-\alpha}}{\zeta x} \mathrm{~d} x\right),
$$

with $\zeta$ in (5.7). The three components of (5.9) correspond to

1. The Laplace transform of $e^{-\delta \tau} X_{t}$.

2. The Laplace transform of a truncated stable $\tilde{T S}$ with truncation level $b w$, and the corresponding Lévy measure is specified in (5.6).

3. The Laplace transform of a compound Poisson $\sum_{i=1}^{N} L_{i}$, with Poisson rate $\frac{\varrho \zeta}{\delta \Gamma(1-\alpha)}$ and jumpsize $\left\{L_{i}\right\}_{i=1,2, . .}$ with density $f_{L_{i}}$ in (5.8).

Based on simulation scheme for truncated stable in Algorithm 4.3 and the decomposition scheme in Proposition 5.2, one could easily sample the truncated stable driven OU process. In 
Figure 8, we illustrate several simulated paths of the truncated stable driven OU processes under parameter settings $\left(\alpha, b, \delta, \varrho, X_{0}\right)=(0.2,0.8,1,1,1),\left(\alpha, b, \delta, \varrho, X_{0}\right)=(0.5,0.8,1,1,1)$, and $\left(\alpha, b, \delta, \varrho, X_{0}\right)=(0.9,1.6,1,1,1)$, respectively. In general, as long as the density of the truncated Lévy subordinator is specified, one could use the marked renewal method to sample the truncated Lévy subordinator and use the decomposition scheme in Qu et al. (2019); Dassios et al. (2017b); $\mathrm{Qu}$ (2019) to sample the corresponding non-Gaussian OU process.

\section{Concluding Remarks}

In this paper, we introduce a new type of Lévy subordinator whose jump sizes are restricted by a certain truncation level. We have derived some important distributional properties of these processes and marked renewal representation which leads to an exact simulation framework in general. In particular, we have developed Algorithm 4.3 to sample the truncated stable process, Algorithm 4.4 to sample the truncated tempered stable process and Algorithm 4.6 to sample truncated stable related process. In addition, we develop an enhanced algorithm, Algorithm 4.5, to improve computational speed for the truncated tempered stable with large tilting parameter. Based on the simulation algorithms for truncated Lévy subordinators, we develop exact simulation schemes for two-sided Lévy processes and truncated Lévy Ornstein-Uhlenbeck processes. Extensive numerical experiments and tests are established in order to demonstrate the accuracy of our results.

\section{Acknowledgement}

The authors would like to thank the Associate Editor and reviewers for many constructive comments that greatly improved the presentation of our results.

\section{References}

Abate, J. and Whitt, W. (1992). The Fourier-series method for inverting transforms of probability distributions. Queueing Systems, 10(1):5-87.

Abate, J. and Whitt, W. (1995). Numerical inversion of Laplace transforms of probability distributions. ORSA Journal on Computing, 7(1):36-43.

Abate, J.and Whitt, W. (2006). A unified framework for numerically inverting Laplace transforms. INFORMS Journal on Computing, 18(4):408-421.

Asmussen, S. and Rosiński, J. (2001). Approximations of small jumps of Lévy processes with a view towards simulation. Journal of Applied Probability, 38(02):482-493. 
Barndorff-Nielsen, O. E. (1998). Processes of normal inverse Gaussian type. Finance and Stochastics, 2(1):41-68.

Barndorff-Nielsen, O. E., Jensen, J. L., and Sørensen, M. (1998). Some stationary processes in discrete and continuous time. Advances in Applied Probability, 30(4):989-1007.

Barndorff-Nielsen, O. E. and Shephard, N. (2001b). Non-Gaussian Ornstein-Uhlenbeck-based models and some of their uses in financial economics. Journal of the Royal Statistical Society: Series B (Statistical Methodology), 63(2):167-241.

Barndorff-Nielsen, O. E. and Shephard, N. (2002). Econometric analysis of realized volatility and its use in estimating stochastic volatility models. Journal of the Royal Statistical Society: Series B (Statistical Methodology), 64(2):253-280.

Barndorff-Nielsen, O. E. and Shephard, N. (2003a). Integrated OU processes and non-Gaussian OU-based stochastic volatility models. Scandinavian Journal of Statistics, 30(2):277-295.

Barndorff-Nielsen, O. E. and Shephard, N. (2003b). Realized power variation and stochastic volatility models. Bernoulli, 9(2):243-265.

Bertoin, J. and Doney, R. A. (1994). Cramér's estimate for Lévy processes. Statistics \& Probability Letters, 21(5):363-365.

Blanchet, J. H. and Sigman, K. (2011). On exact sampling of stochastic perpetuities. Journal of Applied Probability Special Volume, 48A:165-182.

Chi, Z. (2012). On exact sampling of nonnegative infinitely divisible random variables. Advances in Applied Probability, 44(3):842-873.

Dassios, A., Lim, J. W., and Qu, Y. (2019). Exact simulation of vervaat perpetuities. Journal of Applied Probability, 56(1):57-75.

Dassios, A., Qu, Y., and Zhao. H. (2017b). Exact Simulation of tempered stable Ornstein-Uhlenbeck processes: a unified approach. Working Paper, London School of Economics.

Devroye, L. (1968). Nonuniform random variate generation. Springer, New York.

Devroye, L. (2009). Random variate generation for exponentially and polynomially tilted stable distributions. ACM Transactions on Modeling and Computer Simulation (TOMACS), 19(4):18.

Embrechts, P. and Goldie, C. M. (1994). Perpetuities and random equations. Asymptotic statistics (Prague, 1993), pages 75-86.

Furrer, H. (1998). Risk processes perturbed by $\alpha$-stable Lévy motion. Scandinavian Actuarial Journal, 1998(1):59-74.

Furrer, H., Michna, Z., and Weron, A. (1997). Stable Lévy motion approximation in collective risk theory. Insurance: Mathematics and Economics, 20(2):97-114. 
Gaver, J. D. P. (1966). Observing stochastic processes, and approximate transform inversion. Operations Research, 14(3):444-459.

Hepburn, C., Duncan, S., and Papachristodoulou, A. (2010). Behavioural economics, hyperbolic discounting and environmental policy. Environmental and Resource Economics, 46(2):189-206.

Kallsen, J., Muhle-Karbe, J., and Voß, M. (2011). Pricing options on variance in affine stochastic volatility models. Mathematical Finance, 21(4):627-641.

Kanter, M. (1975). Stable densities under change of scale and total variation inequalities. The Annals of Probability, 3(4):697-707.

Kim, Y. S., Rachev, S. T., Bianchi, M. L., and Fabozzi, F. J.(2010) Tempered stable and tempered infinitely divisible GARCH models. Journal of Banking \& Finance, 34(9):2096-2109.

Klüppelberg, C., Kyprianou, A. E., and Maller, R. A. (2004). Ruin probabilities and overshoots for general Lévy insurance risk processes. The Annals of Applied Probability, 14(4):1766-1801.

Li, L. and Linetsky, V. (2014). Time-changed Ornstein-Uhlenbeck processes and their applications in commodity derivative models. Mathematical Finance, 24(2):289-330.

Morales, M. and Schoutens, W. (2003). A risk model driven by Lévy processes. Applied Stochastic Models in Business and Industry, 19(2):147-167.

Nicolato, E. and Venardos, E. (2003). Option pricing in stochastic volatility models of the OrnsteinUhlenbeck type. Mathematical Finance, 13(4):445-466.

Qu, Y. (2019). Simulation on Lévy subordinators and Lévy driven contagion models. PhD Thesis, London School of Economics.

Qu, Y., Dassios, A., and Zhao. H. (2019). Exact Simulation of gamma-driven Ornstein-Uhlenbeck processes with finite and infinite activity jumps. Journal of the Operational Research Society.

Sato, K. and Yamazato, M. (1984). Operator-selfdecomposable distributions as limit distributions of processes of Ornstein-Uhlenbeck type. Stochastic Processes and their Applications, 17(1):73-100.

Schmidli, H. (2001). Distribution of the first ladder height of a stationary risk process perturbed by $\alpha$-stable Lévy motion. Insurance: Mathematics and Economics, 28(1):13-20.

Sozou, P. D. (1998). On hyperbolic discounting and uncertain hazard rates. Proceedings of the Royal Society of London. Series B: Biological Sciences, 265(1409):2015-2020.

Stehfest, H. (1970). Algorithm 368: Numerical inversion of Laplace transforms. Communications of the ACM, 13(1):47-49.

Todorov, V. and Tauchen, G. (2006). Simulation methods for Lévy-driven continuous-time autoregressive moving average (CARMA) stochastic volatility models. Journal of Business \& Economic Statistics, 24(4):455-469. 
Uhlenbeck, G. E. and Ornstein, L. S. (1930). On the theory of the Brownian motion. Physical Review, 36(5):823.

Vervaat, W. (1979). On a stochastic difference equation and a representation of non-negative infinitely divisible random variables. Advances in Applied Probability, 11(4):750-783.

Wang, H. (2017). Robust asset pricing with stochastic hyperbolic discounting. Finance Research Letters, 21:178-185.

Wolfe, S. J. (1982). On a continuous analogue of the stochastic difference equation $x_{n}=\rho x_{n-1}+b_{n}$. Stochastic Processes and their Applications, 12(3):301-312.

Zhang, S. (2011). Exact simulation of tempered stable Ornstein-Uhlenbeck processes. Journal of Statistical Computation and Simulation, 81(11):1533-1544.

Zhang, S. and Zhang, X. (2008). Exact simulation of IG-OU processes. Methodology and computing in applied probability, 10(3):337-355.

Zolotarev, V. M. (1966). On the representation of stable laws by integrals. Selected Translations in mathematical Statistics and Probability, 6:84-88.

Zolotarev, V. M. (1986). One-dimensional stable distributions. American Mathematical Society, Providence, R.I. 